\title{
Stability of exoplanetary systems retrieved from scalar time series
}

\author{
T. Kovács, ${ }^{1 \star}$ \\ ${ }^{1}$ Institute of Physics, Eötvös University, 1117 Budapest, Pázmány P. s. 1A, Hungary
}

Accepted XXX. Received YYY; in original form ZZZ

\begin{abstract}
We propose a novel method applied to extrasolar planetary dynamics to describe the system stability. The observations in this field serve the measurements mainly of radial velocity, transit time, and/or celestial position. These scalar time series are used to build up the high-dimensional phase space trajectory representing the dynamical evolution of planetary motion. The framework of nonlinear time series analysis and Poincaré recurrences allows us to transform the obtained univariate signals into complex networks whose topology carries the dynamical properties of the underlying system. The network-based analysis is able to distinguish the regular and chaotic behaviour not only for synthetic inputs but also for noisy and irregularly sampled real world observations. The proposed scheme does not require neither n-body integration nor best fitting planetary model to perform the stability investigation, therefore, the computation time can be reduced drastically compared to those of the standard numerical methods.
\end{abstract}

Key words: methods: data analysis - planets and satellites: dynamical evolution and stability - celestial mechanics - chaos

\section{$1 \quad 1$ INTRODUCTION}

Since the gravitationally interacting, and therefore nonKeplerian, few-body dynamics can be very complex (Fabrycky 2010), it makes a great deal to investigate the stability of exoplanetary systems containing at least two planets (Rivera \& Lissauer 2001; Armstrong et al. 2015; Batygin et al. 2015; Goździewski et al. 2016; Panichi et al. 2018). Dynamical modeling of multiple planetary systems requires precise initial conditions and system parameters in order to perform reliable n-body numerical integration. The most common methods to constrain the planetary masses and orbital elements are radial velocity (RV) measurements (Laughlin \& Chambers 2001; Rivera \& Lissauer 2001; Tan et al. 2013; Nelson et al. 2014) and transit timing observations, especially their variation (TTVs) (Agol et al. 2005; Holman \& Murray 2005). RV (Laughlin \& Chambers 2006; Pál 2010) and TTV (Nesvorný \& Morbidelli 2008; Nesvorný \& Beaugé 2010; Veras et al. 2011; Deck et al. 2014; Panichi et al. 2018) data sets together provide the input of sophisticated but fairly time consuming statistical methods (e.g. Foreman-Mackey et al. (2013)), which can serve the best fitting models in high dimensional parameter space (with a

^ E-mail: tkovacs@general.elte.hu (TK) certain confidence level) of the underlying planetary dynamics. From the obtained parameters one then can predict the long-term stability of the system.

In addition, low-order analytic and semi-analytic inversion methods often fail close to dynamical degeneracy such as mean motion resonances (MMRs) that are frequent among the known exoplanetary systems (Fabrycky et al. 2014). This shortcoming has been recently avoided by analytic models of transit timing variation for moderate eccentricities and certain range of masses (Agol \& Deck 2016; Deck \& Agol 2016; Hadden \& Lithwick 2016). These methods are suitable to resolve the degeneracy between planetary masses and eccentricities close to low order MMRs.

Nonlinear dynamical systems may often produce chaotic behaviour which means that they are extremely sensitive even for a small perturbation of the initial conditions (Ott 2002; Tél \& Gruiz 2006). This fact makes the application of the above mentioned methods even more problematic or limited to certain conditions. Describing the dynamics of a known deterministic nonlinear dynamical system, when the equations of motion are known, means basically exploring the phase space patterns. This is, however, not the case in the exoplanetary research. In practice, we measure only one signal of the underlying dynamics, and have to obtain the system's behavior from this scalar time series. The ques- 
tion whether the dynamical invariants (such as Lyapunov exponents or other measures of irregularity) of a particular system can be recovered from a single variable data set is, therefore, extremely important.

In this work a widely used nonlinear data analysis method is proposed which is based on the fundamental theorem known as Poincaré recurrence (Saussol et al. 2002). In short, any conservative dynamical system recurs sooner or later to its former states in phase space. Recently, in their report Marwan et al. (2007) showed how the visual interpretation of recurrences, the so-called recurrence plots (RPs) (Eckmann et al. 1987), are quantitatively related to the characteristic of a dynamical system. A generalization of RPs to network representation (Donner et al. 2010; Donner et al. 2011) widely extended the adaptability of recurrences. Application of recurrence network analysis to cutting-edge measurements in exoplanetary research provides a robust and novel method to investigate the stability. Besides the numerical integration of the best fitting planetary models we propose a complementary study to describe the system stability without having any knowledge about the parameters and initial conditions.

The paper is organized as follows. In Section 2, a case study of a two-planet system is presented as a basic dynamical model. The data analysis method is thoroughly described in Section 3. Section 4 examines the application to real exoplanetary data. The main conclusions are drawn in the final section.

\section{THE MODEL SYSTEM}

At this point a simple dynamical model is introduced that ${ }_{11}$ will provide synthetic time series (RV, TTV, and astrometry 115 positions) in order to establish and test the recurrence-based ${ }_{116}$ data analysis method in Section 3. The Sun, Jupiter, Saturn 117 (SJS) subset of our own Solar System has been chosen to be ${ }_{118}$ the basis of the stability analysis.

The well-known semimajor axis-eccentricity $(a, e)$ sta- 120 bility map of the model system is shown in Figure 1. The 121 map is obtained as follows. The barycentric coordinates of 122 the Sun and the two massive planets were integrated by ${ }_{123}$ using the REBOUND-WHFAST routine (Rein \& Tamayo 2015) 124 over 1000 periods of the inner body, i.e. Jupiter. In addi- 125 tion, Saturn's orbital elements cover a grid of $250 \times 250$ ini- 126 tial conditions. That is $a_{\text {Saturn }}=[7.5: 10], \delta a_{\text {Saturn }}=0.01{ }_{127}$ and $e_{\text {Saturn }}=[0: 0.5], \delta e_{\text {Saturn }}=0.002$. Therefore, in these ${ }_{128}$ plots, the semimajor axis and the eccentricity always refer to ${ }_{129}$ Saturn's initial orbital elements in the rest of the paper. In 130 order to characterize the system stability the chaos indica- 131 tor MEGNO (Mean Exponential Growth of Nearby Orbits, 132 Cincotta \& Simó (2000)) is calculated for every set of ini- 133 tial conditions ( $\left.a_{\text {Saturn }}, e_{\text {Saturn }}\right)$. The system is regular for val- ${ }^{134}$ ues about 2 (green) while chaotic for larger MEGNO values 135 (red). It has to be emphasized that most of the chaotic tra- ${ }_{136}$ jectories lead to the disruption of the system. As a result, 137 the integration stops when one of the planets escapes the ${ }_{138}$ system. In this case the value of MEGNO is set to be 8 . The 139 wide green bands penetrating into the chaotic domain cor- ${ }_{140}$ respond to certain MMRs. The rightmost resonance around ${ }_{141}$ $a=9.6$ is the $5: 2$ commensurability between Jupiter and ${ }_{142}$ Saturn.

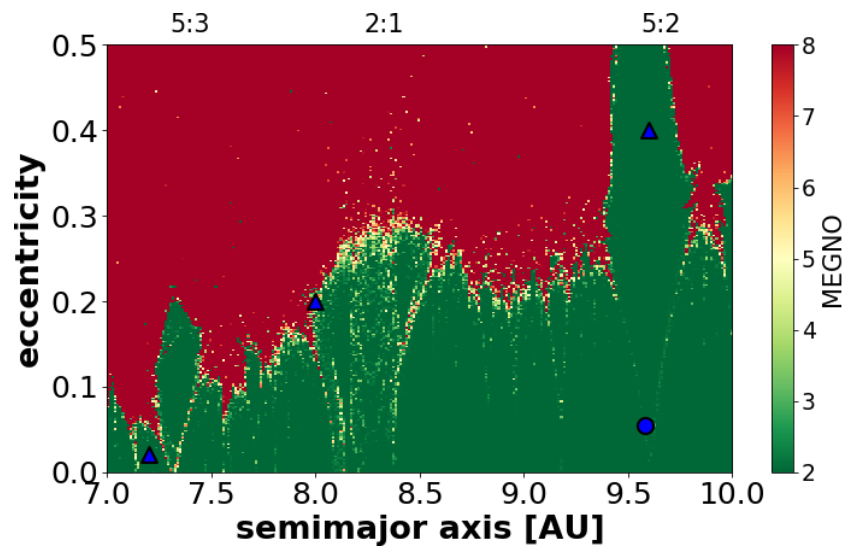

Figure 1. Stability map of Saturn $\left(a_{\text {Saturn }}, e_{\text {Saturn }}\right)$ in SJS system. The stability index MEGNO is color coded according to the color-bar and maximized by 8 . The blue triangles indicate the test data series with different dynamical behaviour as a base of analysis. From left to right: $(7.2,0.02)$ regular non-resonant, (8.0,0.2) chaotic, $(9.6,0.4)$ regular resonant. The blue circle depicts the Saturn's actual position in the $a-e$ parameter plane. The most prominent MMRs are also marked at the top of the panel.

In order to apply the recurrence-based technique to a scalar time series, all numerically computed phase space trajectories are stored and re-used later. This allows one to generate synthetic radial velocity (measured along the $\mathrm{x}$-axis as the line of sight), star position $((\mathrm{x}, \mathrm{y})$ co-ordinates are stored as face on view), and transit time data sets (viewed again from the $\mathrm{x}$-axis) imitating the ideal (noise-free and equidistant) astronomical observations. Some examples are demonstrated in Figure 2. The upper four panels show the RV (a,c) and celestial positions (b,d) of the Sun containing 950 data points. The remaining part of the Figure deals with Jupiter's TTV signal $(\mathrm{e}, \mathrm{g})$ and its mid-transit position (f,h) with a precision of $10^{-5}$ day.

The integration time for the RV data is ca. 1050 orbital periods of Jupiter which means that the sampling frequency is less than the mean motion of the inner planet. TTV signals carry 950 data points similar to those of RV data in order to have the same length of time series in the analysis phase. From now on, these data represent the measurements subject to examination.

The length of the time series is chosen to cover a realistic time frame, i.e around 1000 periods of Jupiter. It might seem to be too long on human time scales but it is not uncommon in currently known, especially tightly packed, exoplanetary systems. For example, 1000 orbital periods of the inner planets (b-e) in the TRAPPIST-1 system corresponds to 1500-6000 days, ca. 4-16 years, the same number of orbital revolution for the Kepler-18 system requires 11-20 years, and even less for Kepler-412. The length of 950 data points can be achieved with the upcoming surveys of the near future.

Here, we take the opportunity and explain a hidden phenomenon between synthetic RV and TTV time series which is not crucial for the analysis but is worth clarifying. Most of the calculations are done for 1050 Jupiter periods in order to obtain the RV signal of the Sun. This data set is then sampled by 950 points equidistantly given a time series to 


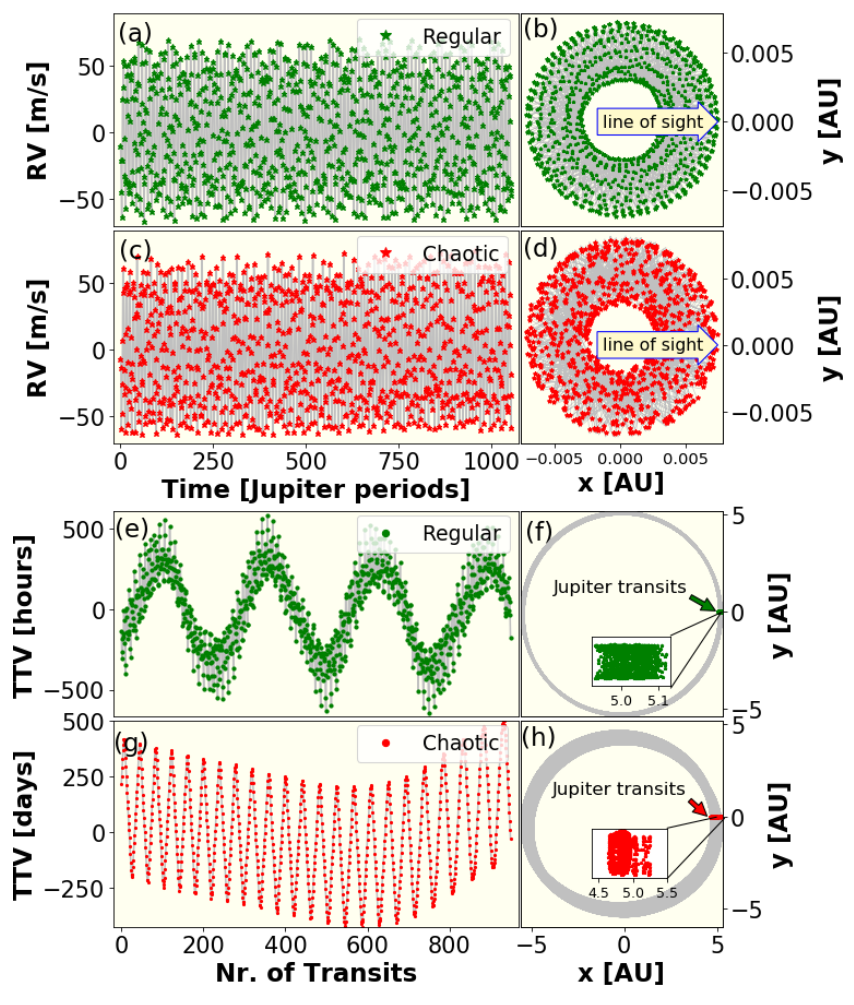

Figure 2. Numerically generated times series for the SJS system. (a) and (c): Radial velocity (RV) of the Sun along the x-axis. (b) and (d): Celestial positions of the Sun in the x-y plane. (e) and (g): Transit timing variation (TTV) of Jupiter. The green and red dots belong to the initial conditions $(7.2,0.02)$ and $(8.0,0.2)$ marked by blue triangles in Fig 1, regular and chaotic respectively. The line of sight is the (positive) x-axis. (f) and (h): Transit positions of Jupiter. The insets highlight the radial "spread" (in AU) of transit positions in the $\mathrm{x}-\mathrm{y}$ plane.

be analysed. This means that every single RV measurement is considered with equal length given above. In contrast, ob- ${ }^{174}$ taining the TTV signals we set the length of the data to 950 Jupiter transits. Clearly, the appearance of transits depends on the dynamics, as well as, on the initial configuration of the system. That is, as the orbital elements change in time ${ }^{176}$ the 950 TTVs can be obtained sooner or later and, there- 177 fore, the time series being analysed might, and actually do, 178 somewhat differ in their length.

\section{METHODS AND TECHNIQUES}

In this section the data analysis method based on phase ${ }^{185}$ space recurrences is discussed in detail. Synthetic time se- ${ }^{186}$ ries are generated as demonstrated in the previous section. ${ }^{187}$ In some cases to have a more realistic scenario noise is added ${ }^{18}$ to data. Where this applies, a precise quantitative descrip- ${ }^{189}$ tion is given in the text. This section is divided into five sub- 190 sections according to the schematic work flow in Figure 3. Subsection 3.1 gives an overview about the phase space reconstruction. The second subsection is devoted to recurrence plots. The link between RPs and recurrence networks is examined in Subsection 3.3. Noisy and unevenly sampled data

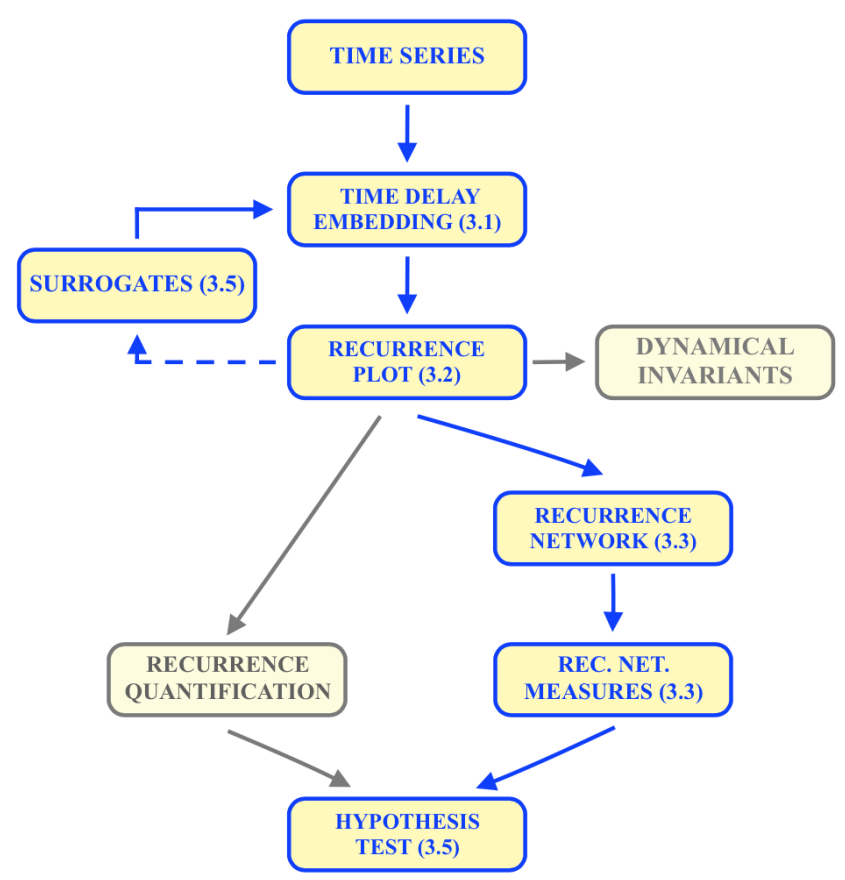

Figure 3. Work flow of the nonlinear time series analysis applied. Dynamical invariants and recurrence quantification are not investigated in present work.

analysis is introduced in Subsection 3.4. Finally, the role of surrogate data analysis and hypothesis testing is demonstrated. This sequence of topics can be thought of as a work flow of the whole procedure, Figure 3 . The reader might feel that this part of the paper is somewhat lengthy, we think, however, that the details presented here are pedagogically necessary to demonstrate how the method works in general before applying it to real data.

\subsection{Phase space reconstruction}

The dynamical analysis of a particular system requires the evolution of phase space trajectories. Since in an experimental setting the observer records the signals in time domain, which means not all relevant components of the state vector is known, a reconstruction of multidimensional information in an artificial phase space is needed. This is possible when the following assumption holds (Semmlow \& Griffel 2014): the variable corresponding to the observable affects the other state space variables, i.e. the variables governing the system's dynamics are coupled. In other words, all hidden variables in the system make some contribution to the measured signal. In this case, a recovery of the phase space trajectory can be done by using an embedding theorem (Takens 1981; Mañé 1981; Packard et al. 1980). In what follows, the method of time delay reconstruction is interpreted.

\subsubsection{Time delay embedding}

Time series $x\left(t_{i}\right)$ is a sequence of $i=1, \ldots, n$ scalar measurements of some quantity $(x)$ depending on the state of 
the system taken at discrete times $(\Delta t)$

$x\left(t_{i}\right)=x(n \Delta t)$.

The reconstructed $m$ dimensional vector reads (Kantz 243 \& Schreiber 2003) then

$\left.x\left(t_{i}\right) \rightarrow \mathbf{x}_{N}=\left\{x\left(t_{i}-(m-1) \tau\right), x\left(t_{i}-(m-2) \tau\right), \ldots, x\left(t_{i}-\tau\right), x\left(t_{i}\right)\right)\right\},{ }^{246}$

(2) 247

where $i=1 \ldots n$ is the length of the original signal, $m$ is the dimension into which the reconstructed vector is embedded, the delay, $\tau$, is the time difference between adjacent components of $\mathbf{x}$. After the delay reconstruction the length of $\mathbf{x}$ is reduced to $N=n-(m-1) \tau$. That is, the components of the reconstructed vector are segments of the original 1D signal delayed by $\tau$. Thus, $\mathbf{x}$ is an $m \times[n-(m-1)] \tau$ matrix. For clarity consider the example in Appendix A.

Takens' embedding theorem (Takens 1981) states that the dynamical properties of a $d$ dimensional attractor can be reconstructed if $m>2 d$ no matter how large the original dimension of the true state space is ${ }^{1}$. Takens also showed that the time lag can have arbitrary value, however, there are practically relevant values.

It should be noted that the reconstructed trajectory is not identical to that one we would have from numerical integration, i.e. when all the components are known. It might differ in shape but preserves the mathematical properties such as topology and Lyapunov exponents.

At this point a natural question might arise. How to find the right embedding dimension and time delay? Since there are many widely accepted methods to choose appropriate values (Kantz \& Schreiber 2003; Ma \& Chong-Zhao 2006) here only those are reported that were used in the current analysis. The embedding dimension $m$ can be obtained by using the concept of false nearest neighbours. The primary goal of this procedure is to find neighbours in embedding space that become not neighbours anymore because the temporal evolution. For a detailed description see Kantz \& Schreiber (2003) and references therein. One method for estimating the time delay $\tau$ is to find the first minimum of the mutual information function (MIF) (Semmlow \& Griffel 2014). MIF can be considered as a nonlinear analog of the autocorrelation function and, therefore, it is more appropriate in this analysis. The TISEAN ${ }^{2}$ software package (Hegger et al. 1999) has been used to calculate the delay parameters through the whole analysis.

At the end of this subsection the parameters of the delay reconstruction $(m, \tau)$ in SJS system are presented. The previously introduced grid of initial conditions $\left(a_{\text {Saturn }}, e_{\text {Saturn }}\right)$ is used, though, the embedding dimension and the time delay is plotted instead of the stability index MEGNO, see Figure 4 . Each grid point in the $\left(a_{\text {Saturn }}, e_{\text {Saturn }}\right)$ parameter plane represents a single time series, Sun's synthetic RV, that has been obtained as interpreted in Figure 2 .

Both maps are similar to those showing the stability index MEGNO in Fig. 1. The sharp border between chaotic

1 It has been shown that fewer dimensions are sufficient for measured data.

2 https://www.pks.mpg.de/ ${ }^{\sim}$ tisean/Tisean_3.0.1/ and regular domains as well as the resonant structures are clearly outlined. This is in a good agreement with the theoretical consideration, the more irregular the dynamics, the less the correlation between its elements. In other words, a motion with increased random elements (i.e. stronger instability) may require larger embedding dimension than ordered motion (Stergiou 2016), see Figure 4(a). In case of a strong gravitational interaction, one of the planets can be ejected from the system. If this happens before the integration is over, the embedding dimension is set to be a large number, $m=100$, and the corresponding initial condition is red. We can conclude from both panels that the optimally determined embedding parameters carry some information about the underlying dynamics. Moreover, the requested time delay indicates a lesser degree of correlation in the dynamics also in agreement with Takens.

\subsection{Recurrence Plots}

Extracting meaningful information from a data set can be easy if regular patterns of the observable appear in time domain. However, when the time series is more complex and no simple rule for its time dependence can be formulated, the representations of certain events might help us to draw some conclusion about the dynamics.

Once the phase space trajectories are available, either the original or the reconstructed one, these representations show up when the state vector returns to a neighbourhood of a point that has already been visited. This phenomenon was first described by Poincaré and is called as recurrence. The related recurrence time statistics became a key concept of dynamical system analysis across many disciplines. These recurrences in phase space can be easily visualized by recurrence plots (RP) originally introduced by Eckmann et al. (1987).

An RP is a very simple method for measuring and visualizing recurrences of a trajectory even in higher dimensions. It can be represented by a two-dimensional matrix $\mathbf{R}$

$R_{i, j}(\epsilon)=\Theta\left(\epsilon-\left\|\mathbf{x}_{i}-\mathbf{x}_{j}\right\|\right), \quad i, j=1 \ldots N$,

where $N$ is the length of the (reconstructed) phase space trajectory, $\Theta(\cdot)$ is the Heaviside step function, $\epsilon$ a tolerance parameter, and $\|\cdot\|$ is a norm. The embedded delay vectors obtained from measured points are $\mathbf{x}_{i}$ and $\mathbf{x}_{j}$ at different time instant, $t_{i}$ and $t_{j}$, respectively. If a trajectory at $t_{j}$ returns to an $\epsilon$ neighbourhood of a point where it was at $t_{i}\left(t_{j}>t_{i}\right)$ then the corresponding matrix element is 1, i.e. recurrence occurs, otherwise 0 . To be more precise

$R_{i, j}(\epsilon)= \begin{cases}0, & \text { when } \epsilon<\left\|\mathbf{x}_{i}-\mathbf{x}_{j}\right\| \\ 1, & \text { when } \epsilon>\left\|\mathbf{x}_{i}-\mathbf{x}_{j}\right\| .\end{cases}$

The matrix $\mathbf{R}$ is symmetric by definition. Plotting the elements of the binary matrix with different colors, one can obtain the RP. Figure 5 depicts four recurrence plots corresponding to two initial conditions marked in Fig. 1 (blue triangles). In panel (a), the lower right triangle (red) depicts the RP corresponding to the reconstructed trajectory from Sun's RV data originating from $\left(a_{\text {Saturn }}, e_{\text {Saturn }}\right)=$ $(8.0,0.2)$, the upper left $($ green $)$ is for $\left(a_{\text {Saturn }}, e_{\text {Saturn }}\right)=$ $(7.2,0.02)$. Panel (b) shows the same obtained from the TTV signals of Jupiter. One can see the differences between the 

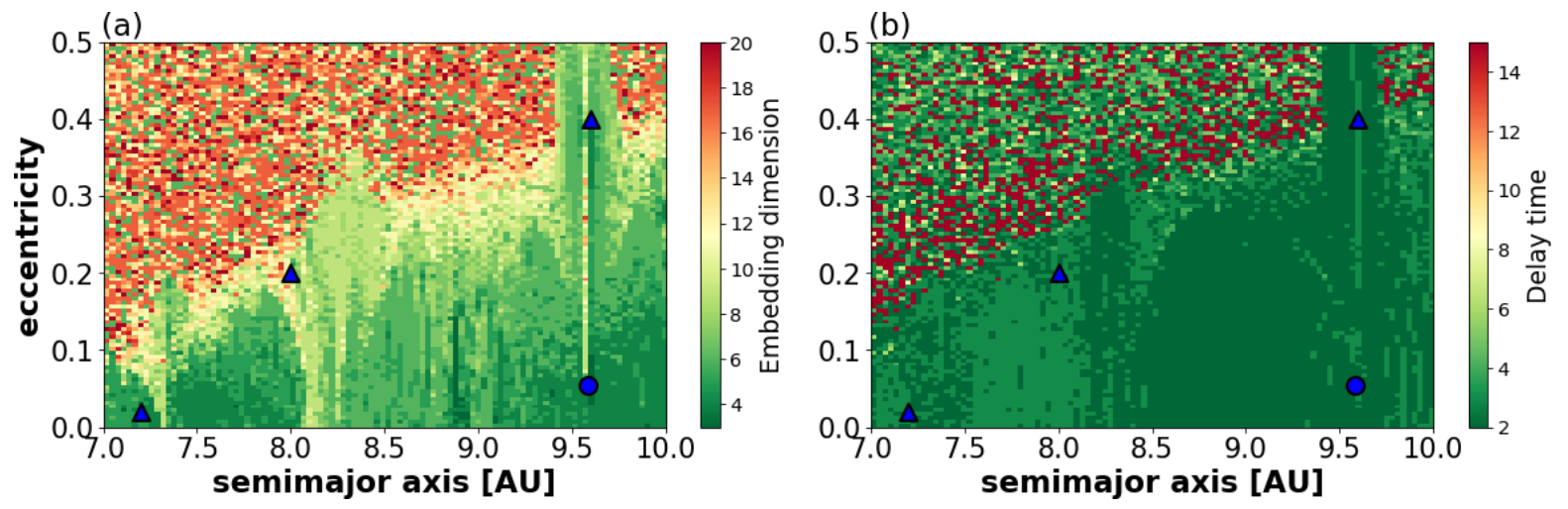

Figure 4. Embedding dimension (a) and time delay (b) dependence for $\left(a_{\text {Saturn }}, e_{\text {Saturn }}\right)$ initial conditions similar as in Figure 1. The resolution is 100x100 points each directions. The synthetic data sets include 950 measurements of Sun's radial velocity during the 1050 Jupiter-periods. Marked points correspond to different dynamical regimes (blue triangles) and the Saturn's actual position (circle) in the $(a, e)$ plane, see also Figure 1. Similar plots can also be constructed from the TTVs and celestial positions, not presented here.

\subsection{Recurrence Networks}

A natural way to analyse complex systems is using complex network theory developed in the last two decades. The fact that nonlinear time series analysis can be used effectively to 322 study complex dynamics and the successful application of 323 networks in various fields stimulated the demand of trans- 324 forming time series into complex networks. Recently, sev- 325 eral different methods have been proposed (Xu et al. 2008; 326 Shimada et al. 2008; Donner et al. 2010). In many cases a 327 network can be describe mathematically as a graph $G(V, E) \quad 328$ (a)

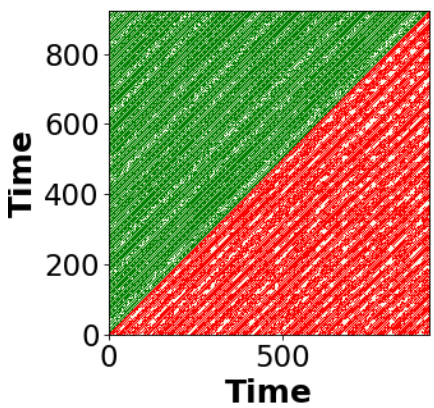

(b)

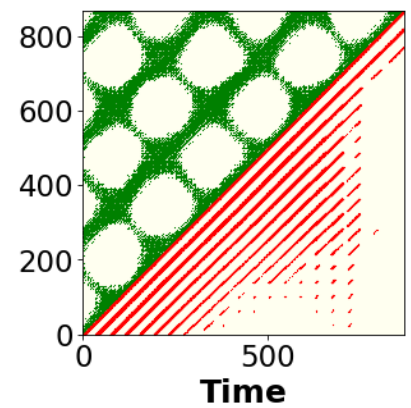

Figure 5. Recurrence plots for two different trajectories in SJS system based on RV data sets (a) and Jupiter's TTV signals (b). Integration time: 1050 Jupiter periods with 950 measurements for RVs and 950 TTVs. The recurrence rate $(R R)$ is fixed to 0.1 . The phase space distance is defined by the maximum norm.

where $V=\{1,2, \ldots N\}$ is a set of vertices and $E \subseteq V \times V$ represents the edges between pairs of vertices. In case of unweighted and undirected networks a symmetric $N \times N$ adjacency matrix $\left(A_{i, j}\right)$ completely describes the graph $G$

$A_{i, j}= \begin{cases}1, & \text { when } i, j \in E, \\ 0, & \text { otherwise }\end{cases}$

In this work only proximity networks, a subclass of networks which are directly related to Poincaré recurrences, will be used. An $\epsilon$-recurrence network (RN) consists of vertices formed by reconstructed state vector in the phase space $\left(\mathbf{x}_{N}\right.$, see Section 3.1.1) connected by edges to other vertices in their $\epsilon$ neighborhood. This definition allows to re-interpret the recurrence matrix $\left(R_{i, j}\right)$ of an RP in the following way

$A_{i, j}=A_{i, j}(\epsilon)=R_{i, j}(\epsilon)-\delta_{i j}$,

where $A_{i, j}$ is the adjacency matrix of a complex network embedded in a phase space, Kronecker delta $\left(\delta_{i j}\right)$ avoids the self-loops in the graph. The matrix $\mathbf{A}$ carries the symmetry properties of matrix $\mathbf{R}$, consequently $\mathrm{RN}$ is a graph with no self-loops and multiple edges. Equation (6) illuminates an alternative view of RPs, namely, the joint proximity observations in phase space can be represented as links in complex 
networks. Moreover, $A_{i, j}$ describes random geometric graphs 377 where the vertices are located in a metric space such as the ${ }_{378}$ reconstructed phase space. The quantitative description of RNs, to be discussed later, provides relevant geometric infor- ${ }^{379}$ mation about the underlying dynamics. Furthermore, recur- ${ }^{3}$ rence network method is not based on temporal correlations or explicit time ordering (Donner et al. 2011). This property of RNs will be extremely useful in case of irregularly sampled data sets.

In order to explore different types of dynamical regimes (quasi-periodic or chaotic) two basic network measures are introduced. Other fundamental quantitative characteristics of complex networks can be found in recent groundbreaking 390 works of Albert \& Barabási (2002); Newman (2003); Boccaletti et al. (2006).

The average path length $A P L$ can be defined as the ${ }^{302}$ arithmetic mean of geodesic distance $\left(d_{i j}\right)$ between all pair of vertices $(i, j)$

$A P L=\frac{2}{N(N-1)} \sum_{i \neq j} d_{i j}$,

where $d_{i j}$ is the minimum number of edges between two 399 vertices. In continuous systems $A P L$ is approximately the 400 length of the trajectory in the phase space, hence, periodic 401 (Zou et al. 2010) orbits are characterized by larger APL than ${ }_{402}$ chaotic ones.

Transitivity $(T R N)$, which is a closely related quantity 404 to clustering, is the relative number of triangles compared ${ }_{405}$ the total number of connected triples of nodes. In contrast 406 to the global clustering coefficient, transitivity gives equal 407 weights to all triangles in the network (Barrat \& Weigt ${ }_{408}$ 2000):

$$
\begin{aligned}
T R N & =\frac{3 \times \text { nr. of triangles in the network }}{\text { nr. of linked triples of vertices }} \\
& =\frac{\sum_{i, j, k} A_{j k} A_{i j} A_{i k}}{\sum_{i, j, k} A_{i j} A_{i k}\left(1-\delta_{j k}\right)} .
\end{aligned}
$$

After the mathematical definitions of RN-related quantities, let us apply them to the synthetic data sets obtained from the model systems SJS. The average path length and transitivity has been calculated over the stability map $\left(a_{\text {Saturn }}, e_{\text {Saturn }}\right)$. Figure 6 summarizes the results based on the RV of the Sun and the TTV of Jupiter.

At a glance, it is obvious that all four panels show the structure of Figure 1. The chaotic and regular parts can be easily distinguished. Nevertheless, the details around MMRs and at the edge of chaos also match the texture. Before going into the further details, we should highlight some important results of previous studies. Zou et al. (2010) and Zou et al. (2016) investigated continuous and discrete dynamical systems by means of recurrence network technique. They found the following specific features:

(i) Transitivity takes larger value for regular orbits and 43 lower value for chaotic ones both in continuous and in discrete systems.

(ii) However, the average path length shows different behavior. For discrete systems, the $A P L$ of a periodic orbit is smaller than that of a chaotic one. In contrast, for continuous systems, periodic orbits are characterized by larger average path length than chaotic ones.

Now, we can analyse the panels in Figure 6 in more details. Let us concentrate on the left column first where the color maps depict the recurrence network measures APL and TRN based on the Sun's radial velocity. The results we see here are, loosely speaking, based on a continuous dynamical system. Since the time series are obtained from the RV of the Sun which is a real component of the phase space trajectory. Panel (a) demonstrates that regular motion accomplishes larger APL while chaotic behaviour comes with smaller values. This also applies for the measure transitivity in good agreement with point (i) above.

The panels (b,d) belonging to TTVs of Jupiter, i.e. the network measures acquired from the transit times of the larger planet, can be interpreted somewhat differently. We should see that transit times either of Jupiter or Saturn do not represent any component of the phase space trajectory. They are based on special configurations when the planet passes in front of the star. This situation can be thought of as a discrete map ${ }^{3}$ rather than a continuous trajectory, which plays the a crucial role in our following argument. If we consider the TTV signal as a map-like description of a continuous dynamical system, the information in panel (b) is consistent with (ii). Namely for discrete systems $A P L$ is smaller for regular motion. Besides, the landscape of the ( $\left.a_{\text {Saturn }}, e_{\text {Saturn }}\right)$ map shows extremely fine details of the stability regions and MMRs (recall Figure 1). It is clear that measure $A P L$ is capable of distinguishing regular and chaotic behavior based on measurable quantities such as RV and TTV in a synthetic two-planet system.

Scrutinizing panel (d) the overall picture is promising since it is almost identical to panel (b). However, one can observe that the $T R N$ values for regular motion are clearly less than those for chaotic orbits. This situation completely contradicts point (i) above. To understand the discrepancy several control computations have been done including different embedding parameters, longer integration time and also longer time series with more data points. However, we always find the same situation, namely regular motion is characterized by lower transitivity. Donner et al. (2010) and also Zou et al. (2010) underline that for discrete systems the RN breaks down into disjoint components around periodic orbits since they appear as finite sets of points in the phase space. Thus the transitivity tends to 1 . This is, however, not the case in higher dimensions. Because the periodic orbits and trajectories nearby are not restricted to small domains of the phase space anymore. Consequently, the regular trajectories can explore regions as large as the chaotic ones in the phase space. For more details, we draw the readers attention to Appendix B where we demonstrate the effect discussed above by using coupled standard maps as high dimensional discrete Hamiltonian systems. Based on the previous argument, we point out that the $\mathrm{RN}$-measure

3 Well-known maps in dynamical systems theory are the stroboscopic and Poincaré maps. However, transit times do not satisfy the required conditions for these concepts. 

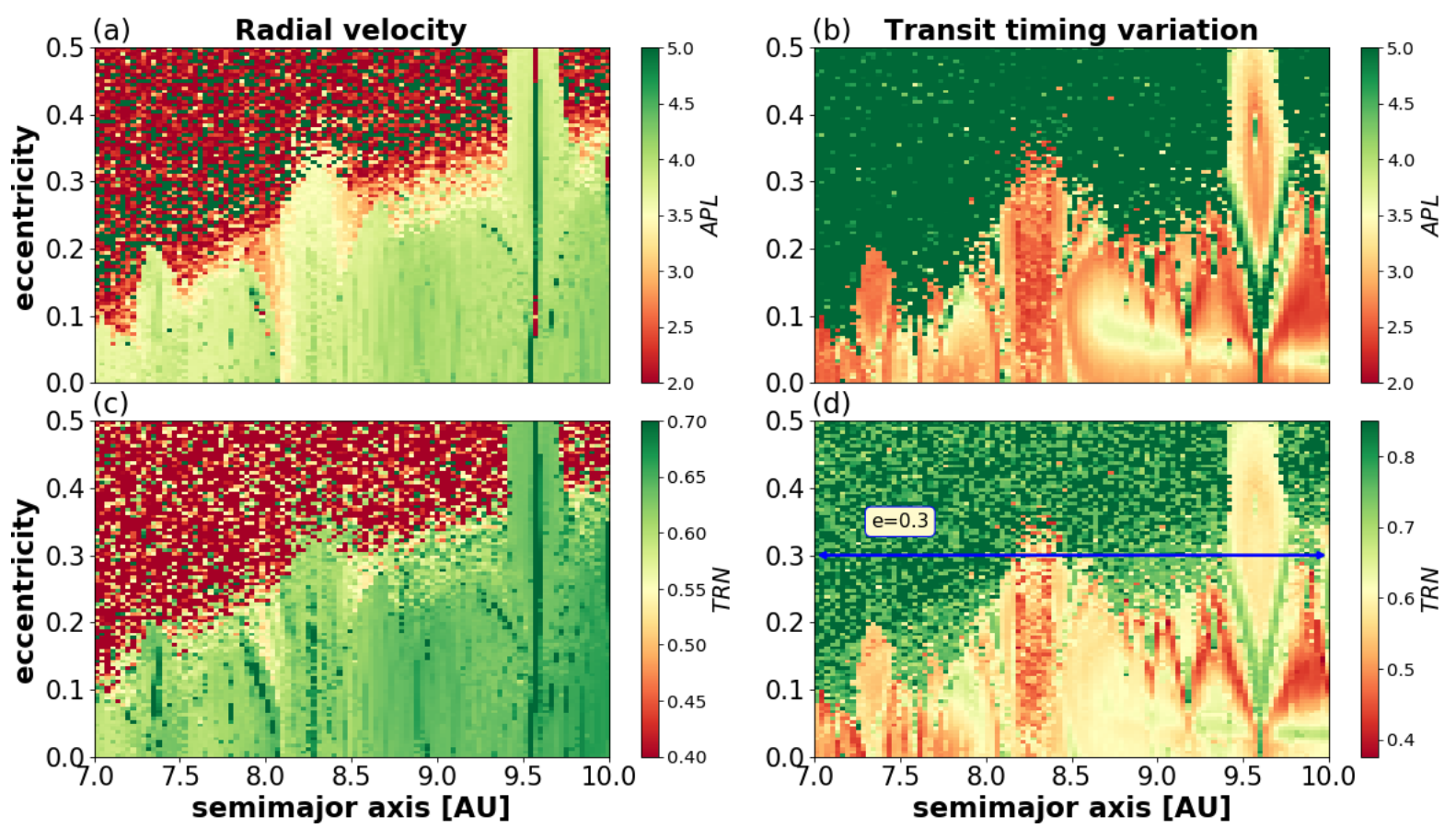

Figure 6. Color maps of two RN measures $A P L$ and $T R N$ over the initial condition grid $\left(a_{\text {Saturn }}, e_{\text {Saturn }}\right)$. On the left the base of the analysis is the Sun's RV while on the right it is Jupiter's TTV signal. Every time series contains 950 data points. The $e_{\text {Saturn }}=0.3$ line is marked in panel (d) in order to guide the eye in Figure 7. To calculate various RN measures the publicly available PYUNICORN (http://www.pik-potsdam.de/ ${ }^{\sim}$ donges/pyunicorn/) package has been used (Donges et al. 2015).
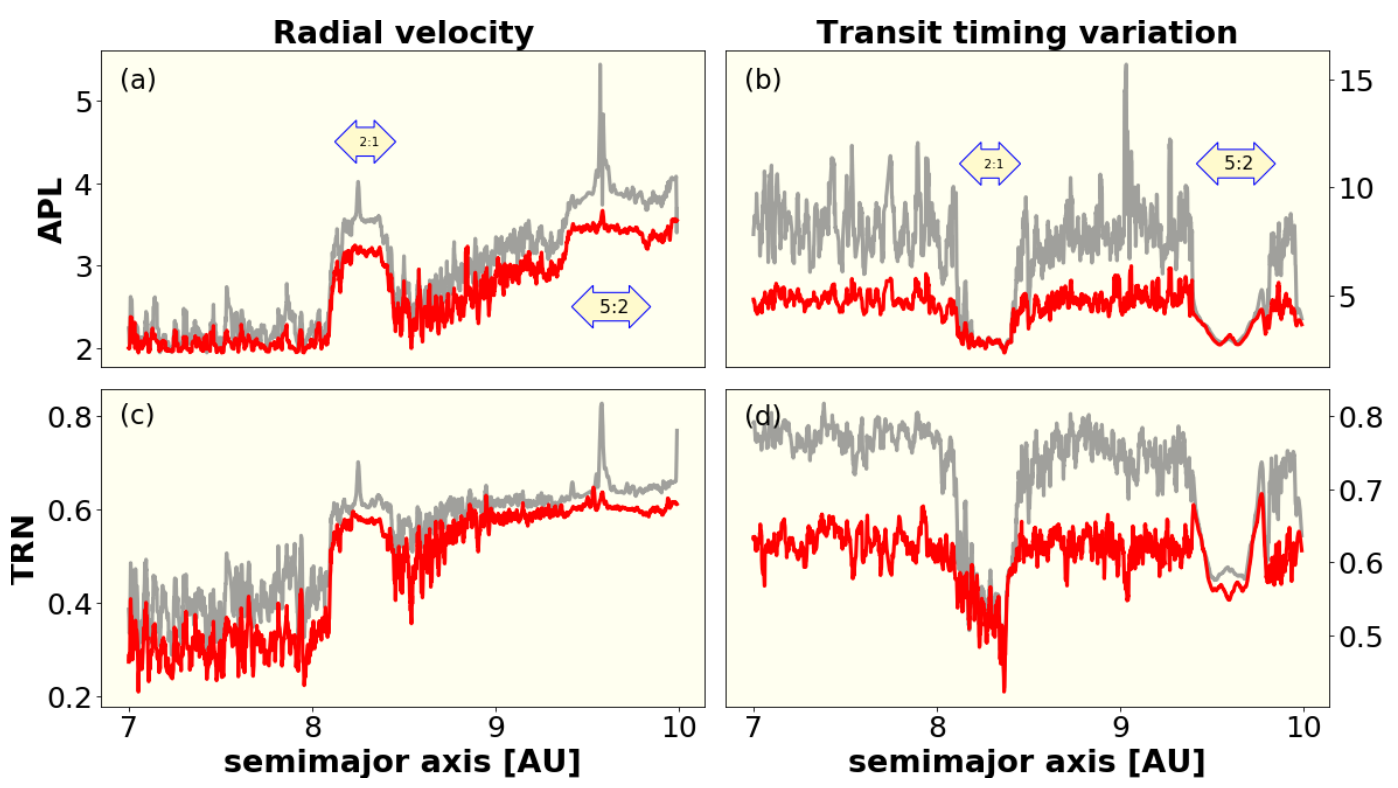

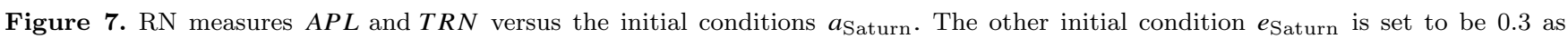
indicated in Fig. 6. The two curves represent the synthetic (gray) and noisy-gappy (red) data sets, respectively.

4 transitivity (TRN) provides smaller value for regular dynam- 437 ics and larger value for chaotic motion when analysing the three body problem from discrete dynamical systems point ${ }^{43}$ of view, i.e. when the phase space reconstruction is based on ${ }^{439}$ the TTVs.

\subsection{Noisy time series and missing data points}

Up to now the analysis has been performed on numerically generated synthetic time series where the measurements evenly sample the exact noiseless calculations. However, to 441 demonstrate the robustness of $\mathrm{RN}$ analyses against noise and 442 possible missing data points the theoretical calculations are 443 modified as follows. 
dard deviation equal to one, is added to the original time ${ }^{505}$ series. The amplitude of the noise is chosen to be $15 \%$ of the ${ }^{506}$ amplitude of the original signal. Furthermore, in order to 507 imitate astronomical observations (even those obtained by 508 space based detectors) a certain amount (20\%) of randomly 509 selected data points have been extracted from the original 510 time series. More precisely, in the first stage the center of an 511 interval is randomly defined along the data set, then, in the 512 second stage, the length of this interval is chosen again ran- 513 domly. The data points falling in the interval are removed 514 from the signal. This procedure is repeated until the desired ${ }^{515}$ percentage of the missing data is achieved.

In Section 3.1 time delay embedding was established. ${ }^{517}$ This type of reconstruction requires uniformly sampled time ${ }^{518}$ series which is not fulfilled when dealing with scanty data ${ }^{519}$ set. It has been recently shown by Lekscha \& Donner (2018) ${ }^{520}$ that a cubic spline interpolation of the original data back to ${ }^{521}$ uniform time series followed by the classical time delay em- ${ }^{522}$ bedding provides reasonable good phase space reconstruc- ${ }^{523}$ tions. According to this, we use in our analysis time delay ${ }^{524}$ embedding on cubic splined data sets.

Figure 7 illustrates the RN measures APL and TRN in 526 the noisy SJS model. In order to save computation time ${ }^{527}$ only one section (see the blue arrow in Figure 6d) of the ${ }^{528}$ $\left(a_{\text {Saturn }}, e_{\text {Saturn }}\right)$ plane has been investigated. Each panel ${ }^{529}$ contains the results based on the synthetic time series (gray) ${ }^{530}$ as well as the noisy and non-uniformly sampled data (red). ${ }^{531}$ Basically the gray curves correspond exactly to the values in 532 Figure 6. The red dots follow the gray structure but the con- ${ }^{533}$ trast is somewhat weaker. That is, the effect of the presence ${ }^{534}$ of noise and missing data points results in smaller difference ${ }^{535}$ between regular and chaotic values of RN measures. Nev- ${ }^{536}$ ertheless, the ordered motion corresponding to MMRs (2:1 ${ }^{537}$ and 5:2) is still perfectly detectable. One can also observe ${ }^{538}$ that RN measures for RV data behave somewhat different ${ }^{539}$ than that of TTV, especially between the two MMRs. And ${ }^{540}$ also the same applies here what has been discussed earlier, ${ }^{541}$ namely, lower values of $T R N$ correspond to regular motion in TTV signals.

We have demonstrated that recurrence network measures are precise and convenient tools to analyse regular and ${ }_{543}$ chaotic motions in gravitational three body problem. The ${ }_{544}$ method is also acceptable in real world examples when the ${ }_{545}$ signal is loaded with noise and the sampling is not perfectly 546 uniform. Nevertheless, it should be emphasized that the ob- 547 tained numbers $(A P L, T R N)$ are relative values even though ${ }_{548}$ they distinguish the different types of motion correctly. This ${ }_{549}$ means, for example, that the average path length for regu- 550 lar dynamics is smaller than for chaotic (in the case of discrete systems). Therefore, the question arises naturally how one can decide from one single scalar time series whether it comes from a chaotic/regular dynamics when we do not have any objective reference point. The answer is given by surrogate data analysis described in next section.

\subsection{General principles of surrogate tests}

Surrogate tests are examples of Monte Carlo hypothesis tests (Theiler et al. 1992; Theiler \& Prichard 1996) applied for testing nonlinearity in a time series. The basic idea is that a nonlinear observable $\lambda_{0}$ is computed from the data and then one has to decide whether $\lambda_{0}$ suggests that the data are ${ }_{551}$ nonlinear. First, a null hypothesis is taken we want to test, say, the data come from linear processes. Then a number of artificial data sets are generated which are consistent with the null hypothesis. This means they have linear properties similar to those of the original data but nonlinearities are removed. In practice, the newly generated surrogates preserve some properties of the original signal (mean, variance, power spectra). Having the ensemble of surrogate time series discriminating statistics are performed, i.e. the nonlinear observables $\lambda_{i}$ are also computed for the surrogates and then they are compared with the original one. If the value of discriminating statistics $\lambda_{0}$ from the original time series does not fall within the distribution of the discriminating statistics of the surrogates, the null hypothesis should be rejected. Otherwise, the signal does not contain nonlinearities.

To quantify this process, we can use hypothesis testing (Kantz \& Schreiber 2003; Schreiber \& Schmitz 2000). If we have a good reason to suppose that the distribution of $\lambda_{i}$ is Gaussian, then the mean and standard deviation define the significance which can be used to construct a desired significance level of inference. However, in general, the distribution of discriminating statistics of surrogate data set is not normal, and, therefore, using a rank-based test instead is a better choice. Suppose $N$ surrogate time series are generated and $\lambda_{i}$ are the calculated nonlinear measures of $i$ th $(i$ $=1 \ldots, N)$ data set. Let $\lambda_{0}$ be the discriminating statistics for the original signal. There are $N+1 \lambda \mathrm{s}$. Now, all these discriminating statistics are ranked in an increasing order. If the original signal was generated by a linear process, the chance that $\lambda_{0}$ is the smallest will be $1 /(N+1)$. According to the rank-order strategy, the null hypothesis is rejected if $\lambda_{0}$ is the smallest among the $(N+1) \lambda \mathrm{s}$. This will give a false rejection if $\lambda_{0}$ being smaller than other $\lambda_{i}$ by chance, which occurs with probability $1 /(N+1)$. That is, if we want to have a false rejection with $95 \%$ significance (2 'sigma'), 19 surrogate time series have to be generated in case of one-sided test.

\subsubsection{Pseudo-Periodic Twin Surrogates (PPTS)}

Since our method is based on recurrence network analysis, and the exoplanetary observables show mostly quasiperiodic and chaotic behavior, we use, through our analysis, the PPTS method to generate surrogate time series. The PPTS algorithm uses the phase space structure and RP representation to generate surrogates.

In what follows, we give the algorithm of the PPTS (Carrión \& Miralles 2016).

(1) Construct the RP of the original signal using Equation (3) with predefined $R R$ and identify the twin points. The larger the $R R$, the more twins. We found the $R R=0.1-0.15$ is adequate in order to find twins.

(2) Randomly choose an initial condition $i_{0}$ and set $i=i_{0}$. Initialize $n=1$.

(3) If there is a twin point for $\mathbf{x}(i)$, make the next point of the surrogate $\mathbf{x}_{\mathrm{S}}(n)=\mathbf{x}(j)$, where $j$ is randomly chosen among the twin points with probability $1 / T$ (here $T$ is the number of twins for the state $\mathbf{x}(i))$. Let $i=j$ and $n=n+1$. 
(4) If there is no twins for $\mathbf{x}(i)$, choose a neighbor $\mathbf{x}(j)$ from all of the elements of the phase space representation with probability

$$
P(\mathbf{x}(j)) \propto \exp \frac{-\|\mathbf{x}(i)-\mathbf{x}(j)\|}{\rho},
$$

where $\rho$ is the noise radius (Small et al. 2001). Set the next point of the surrogate $\mathbf{x}_{\mathbf{S}}(n)=\mathbf{x}(j)$. Let $i=j$ and $n=n+1$.

(5) Repeat from (3) and (4) until $n=N$.

The crucial step in the PPTS algorithm above is how to choose the noise radius $\rho$ in Eq. (9). When $\rho$ is too small, the original signal and the surrogate are identical, while in case of too large noise radius the generated surrogates will be uncorrelated random points. Small et al. (2001) suggest a careful selection method for $\rho$ when the noise level is fine tuned so that the intercycle dynamics are destroyed but intracycle dynamics are preserved. We propose a different method to find the desired noise radius. The base of our alternative is to measure the similarity between two time series, called dynamic time warping (DTW). Here, we only use the outcome, i.e. the value of $\rho$, of the DTW procedure (a detailed description and example can be found in Appendix C).

As mentioned above the PPTS method is strongly connected to the recurrences and the underlying phase space structures. Since recurrence plots determined from PPT surrogate data behave differently for regular and chaotic time series compared to the RPs obtained from the original signal, one can test the null hypothesis that the observed time series is consistent with quasi-periodic orbit.

Now, let us consider several examples from the model system SJS to see how PPTS works in practice. We pick up two initial conditions from the $\left(a_{\text {Saturn }}, e_{\text {Saturn }}\right)$ parameter plane one regular and one chaotic as usual. First, we deal with RV data. Figure 8(a) and (b) portray the RV time series, i.e. Sun's velocity component $v_{x}$, for initial conditions $\left(a_{\text {Saturn }}, e_{\text {Saturn }}\right)=(7.2,0.02)$ and $\left(a_{\text {Saturn }}, e_{\text {Saturn }}\right)=$ (8.0, 0.2), similarly as in Fig. 2. These two initial conditions are also marked as blue triangles in Figure 1. Both data sets contain 950 points, the difference to those in Figure 2 is that current time series are cubic splined (due to missing observations) with additional noise. According to the description above we generated 100 surrogates for regular as well as chaotic time series, respectively in order to achieve $99 \%$ significance in hypothesis test. Panels (c) and (d) illustrate one pair of the corresponding pseudo-periodic twin surrogates.

Having the surrogate data sets we can compare the RN measure of the original signal and those obtained from PPTSs. We focus on APL and TRN as before. The bottom four panels, Figure $8(\mathrm{e})-(\mathrm{h})$, show the relation of these values. In each plot the red solid line represents the $A P L$ and $T R N$ values of the original signal. The blue circles correspond to the $A P L \mathrm{~s}$ and $T R N \mathrm{~s}$ for 100 different PPTS data sets. The rank-based test reveals that in the regular case both $A P L$ and $T R N$ fall into the zoo of surrogate RN measures. In contrast, when the dynamics is chaotic the original measures are located well outside the set of surrogate
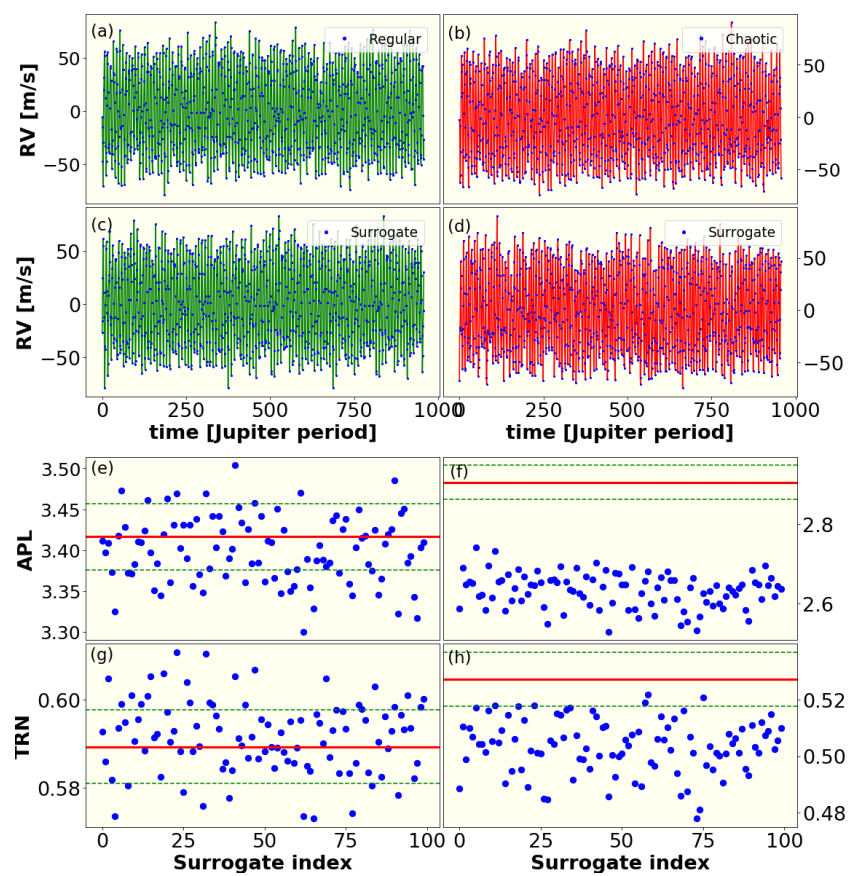

Figure 8. Hypothesis test based on the RV signal in the SJS system. Left: Regular dynamics - original time series (a), one of the surrogate data sets (c), different recurrence network measures $(A P L, T R N)$ compared to the same characteristics calculated from PPTSs. The noise radius appearing in the PPTS algorithm is $\rho_{\text {stable }}=0.202$. Right: Chaotic dynamics - panels match those on the left. The green dashed lines denote the \pm 1 standard deviation of blue data points around the red line ( $\mathrm{RN}$ measures of the original signals). $\rho_{\text {chaotic }}=0.252$.

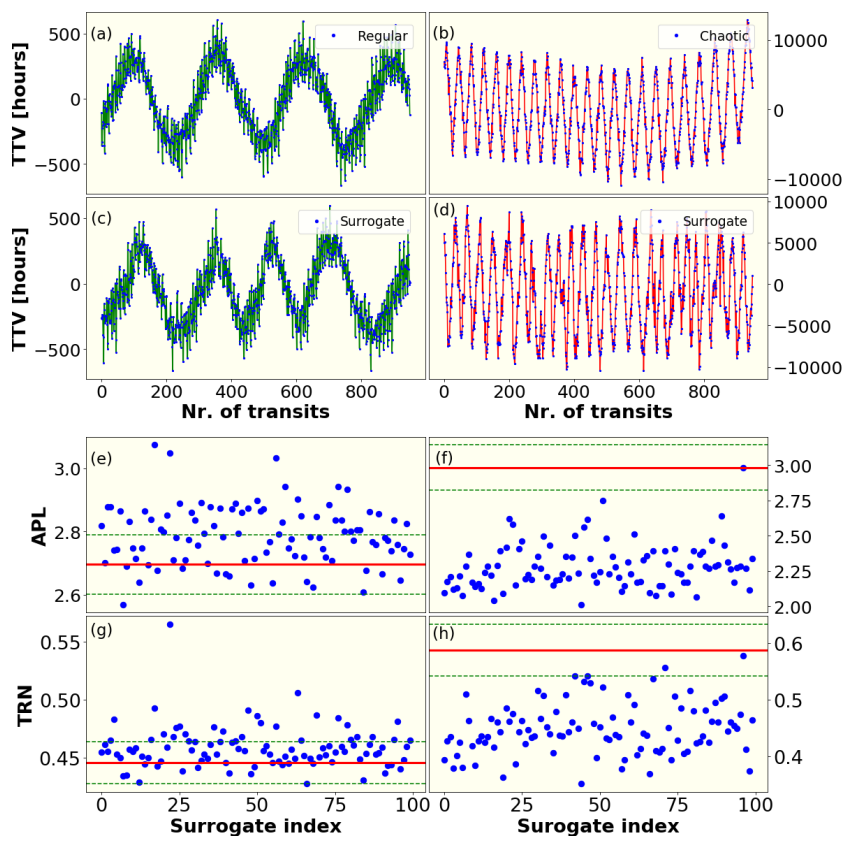

Figure 9. Hypothesis test exploration of (Jupiter) TTV signal. The panels have the content as in Figure 8. $\rho_{\text {stable }}=0.089$ and $\rho_{\text {chaotic }}=0.13$, see Appendix C. 

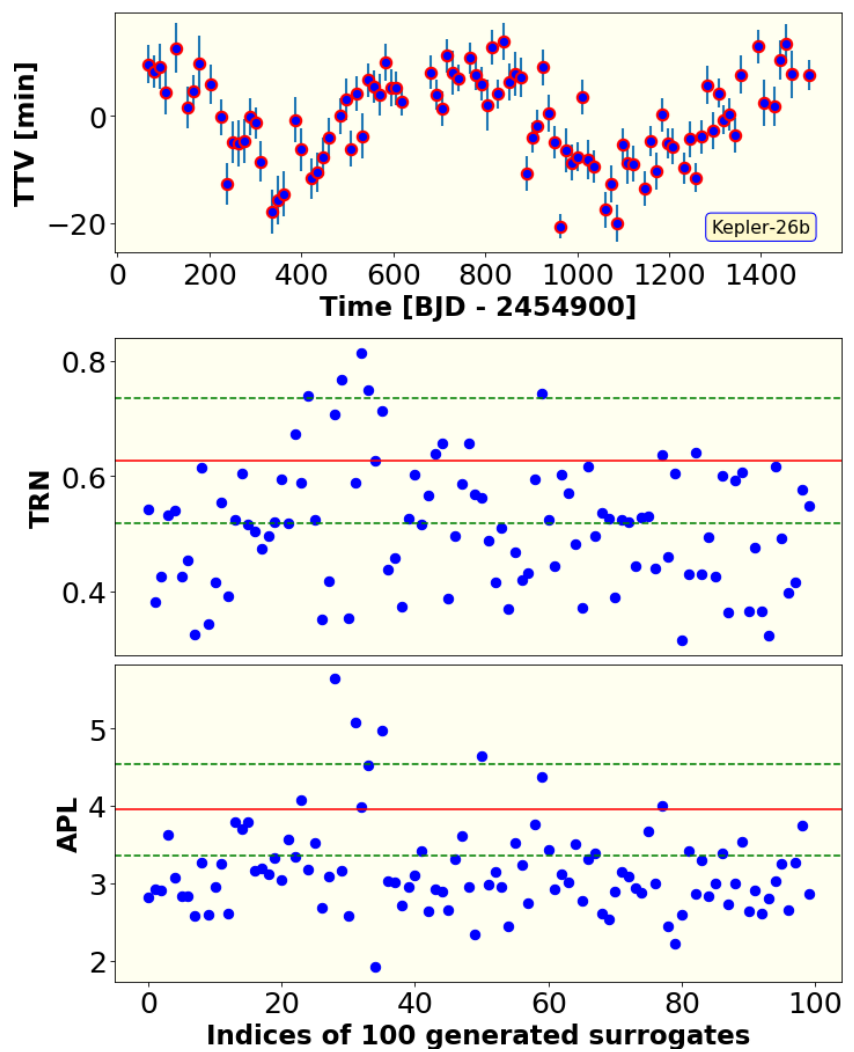

Figure 10. TTV signal (top) and hypothesis test (middle and bottom) of Kepler-26b. The horizontal solid line represents the $T R N=0.627$ and $A P L=3.951$ for the original time series, while the dashed lines show the \pm 1 standard deviation $(\rho=2.076)$ of the same measures calculated from 100 PPTSs (circles).

points. That is, in the later case we can reject the null hypothesis, according to which the original signal comes from quasi-periodic motion.

One can accomplish the same analysis on TTV data sets as well. Figure 9 depicts the hypothesis test for the same two ${ }_{627}$ orbits based the TTV signal of Jupiter ${ }^{4}$. We call again the readers' attention that 950 transits cover slightly different time spans for different types of motion.

\section{APPLICATION TO EXOPLANETARY SYSTEMS}

The results presented in previous sections are based on numerical integration of a well-defined planetary system including the Sun, Jupiter, and Saturn. Our RN analysis shows that 950 data points either RV or TTV measurements (in ${ }^{639}$ case of Jupiter) are enough to carry out the stability investi- ${ }^{640}$ gation of the system. Next we want to apply the whole pro- ${ }^{641}$ cedure to real exoplanetary systems that are known in the ${ }^{642}$ literature. As always, the amount and quality of the acquired ${ }^{643}$

4 The method works well for Saturn's TTV too.
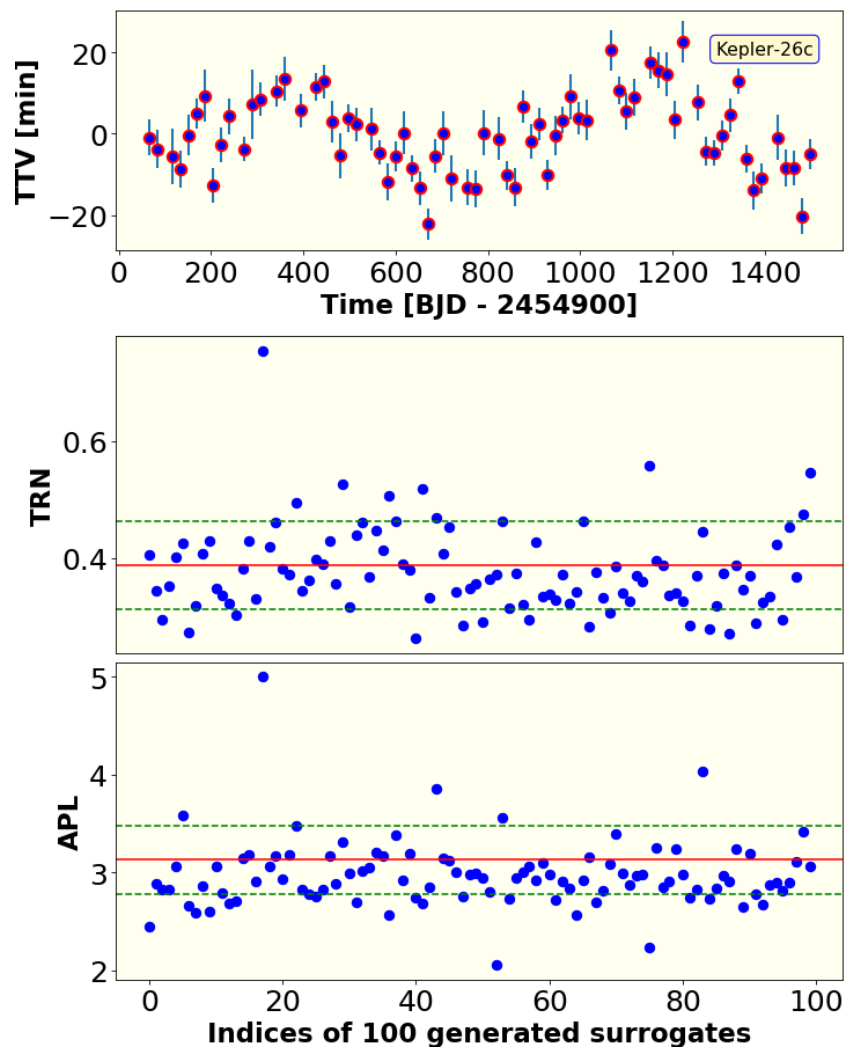

Figure 11. Top: Kepler-26c TTV. Middle-bottom: Hypothesis test for Kepler-26c. $T R N=0.387, A P L=3.133, \rho=2.977$.

data is extremely important, we try to analyse the best public data sets. Thus, we decide to use only space-based TTV signals, e.g. Kepler data, since the available RV measurements about two-planet systems contain a small number of data points and are very sparse in time.

\subsection{Data}

After 17 quarters the Kepler satellite finished its original mission and collected more than 69,000 transits for 779 KOIs with high signal to noise ratio (SNR). The most interesting systems with significant long-term TTVs have been pilled up and published in a catalog (ftp://wise-ftp.tau.ac.il/ pub/tauttv/TTV/ver_112) (Holczer et al. 2016) in order to make the light curves more usable for further research. We limited the choice of possible systems to those presented in Holczer et al. (2016) and their stability analysis can be found in a recent paper of Panichi et al. (2018) for comparison.

\subsubsection{Four-planet system: Kepler-26}

The stability analysis of this planetary system (Steffen et al. 2012) is based on the TTV signals of two super-Earths (out of the confirmed four by Jontof-Hutter et al. (2016)) being ind time series has $17 \%$ missing transits in the available fhen after the phase space reconstruction the noise radius has been obtained as, $\rho=2.076$. Having the value of $\rho$ we can generate the surrogates and perform the 

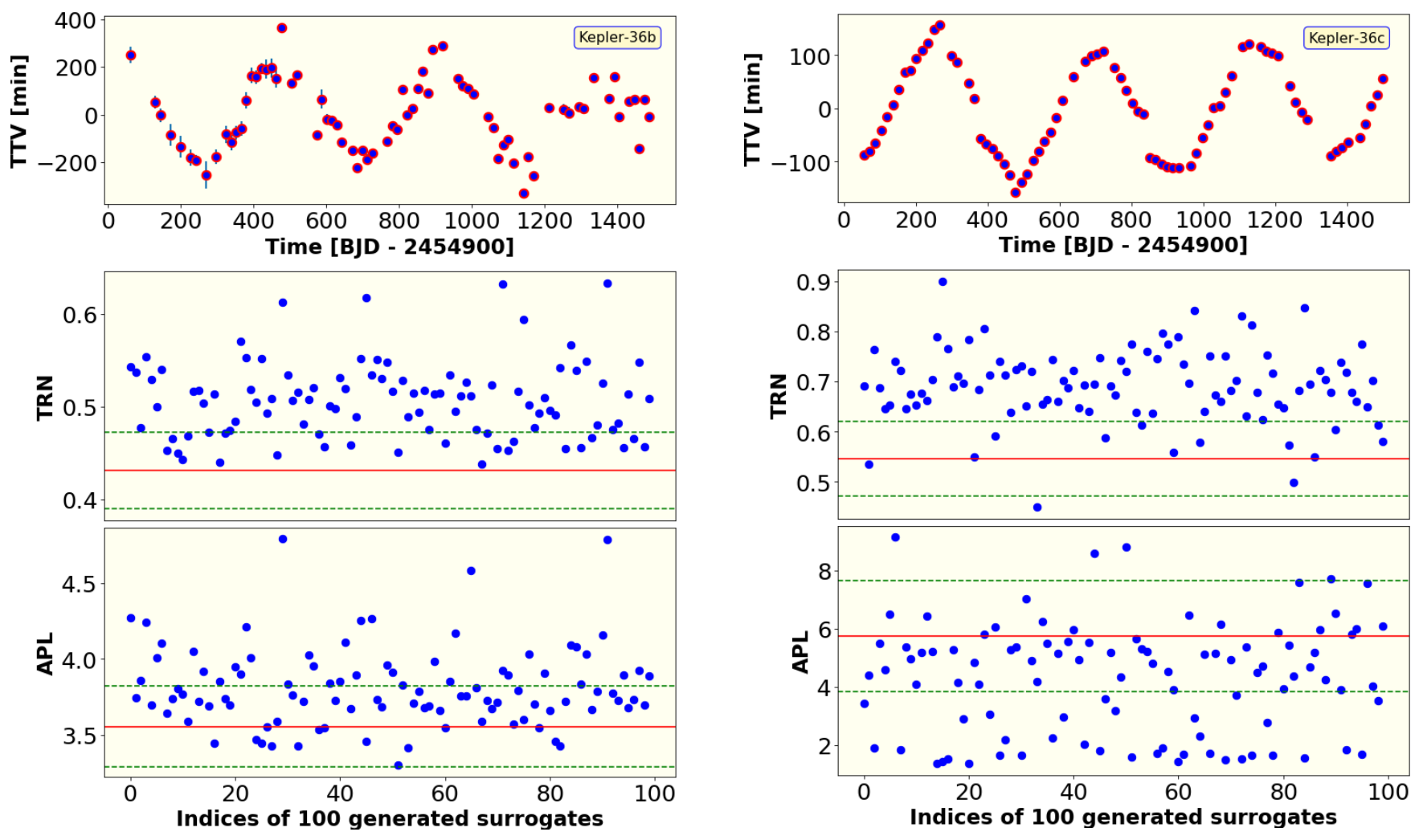

Figure 12. TTV of Kepler-36b (missing data $13 \%$ ) and hypothesis test. The panels have the content as in Figure 8. $T R N=0.430$ , $A P L=3.557, \rho=37.177$. Credit: Kovacs (2019).

hypothesis test. Figure 10 shows the TTV signal and the results of the hypothesis test for the inner planet, Kepler26b. The two bottom panels portray transitivity $(T R N)$ and average path length $(A P L)$ of recurrence network (red solid lines). The same measures of the 100 surrogates (blue circles) encompass those coming from the original signal, the null hypothesis can be accepted, i.e. the observed time series is produced by quasi-periodic dynamics.

The same applies to Kepler-26c. The missing transit events give a signal of $14 \%$ of the whole covered time span. Based on the hypothesis test one can conclude that the planetary dynamics shows regular motion, see Figure 11.

4.1.2 Two-planet system: Kepler-36

The Kepler-36 system (Deck et al. 2012) has one of the ${ }^{684}$ largest TTV signals among the known planetary configu- ${ }^{68}$ rations. Based on this fact, the dynamical analysis, derived ${ }^{686}$ from TTVs of two planets, assisted to explore a complex be- ${ }^{687}$ haviour in this extrasolar planetary system. A great success ${ }^{68}$ of the exploration of Kepler-36 is the emergence of stable ${ }^{60}$ chaos. ${ }^{5}$ In our analysis the hypothesis test shows no consis-

A phenomenon related to a short dynamical life time, in con trast to the orbital elements that indicate long regular motion.

87

Figure 13. TTV of Kepler-36c (missing data $30 \%$ ). Hypothesis test. $T R N=0.546, A P L=5.75, \rho=20.381$. Credit: Kovacs (2019).

tent results. Neither for the two component of the system nor for the different network measures. As one can see, the original measure of $T R N$ appears to be the lowest one in the rank based test middle panel of Figure 12. This means that the null-hypothesis can be rejected, i.e. the original signal comes with $99 \%$ level from chaotic dynamics rather than quasi-periodic. The other measure, $A P L$, shows the opposite (bottom panel) yielding that we can expect a regular motion. This is, however, not the case for Kepler-36c (Figure 13). Kepler-36c appears to be stable for both $T R N$ and $A P L$ in hypothesis test. In fact, most of the surrogate transitivity values are above the $T R N$ of the original time series (middle panel of Fig. 13). Still following the previous rules we accept the null-hypothesis. Although, three out of four tests characterize the system as a regular one, the discrepancy definitely shows more uncertainty. What we can conclude based on the SJS model system and its hypothesis test, see Figure 9 is that the motion taking place in Kepler-36 is very close to the resonance border just like the chaotic $\left(a_{\text {Saturn }}, e_{\text {Saturn }}\right)=(8.0,0.2)$ pair in SJS system. The stable chaos scenario suggested by Deck et al. (2012) completely overlaps with the stickiness effect appearing at the border of regular domains (MMRs in celestial mechanics) in dynamical systems (Tsiganis et al. 2000). In addition, Panichi et al. (2018) also found that the system (Kepler-36) is very close to the border of the 7:6 MMR. 


\subsubsection{Two-planet system: Kepler-29}

The Kepler-29 system (Fabrycky et al. 2012) harbours two super-Earth planets on tightly separated resonant orbits. This indicates a fairly large TTV signal plotted in Figs. 14 and 15 upper panels. Unfortunately, the data is very sparse compared to other time series in the catalog. The 1500 days run contains only 93 valuable transit measurements (from possible 108) producing a signal with 32-36\% missing points. Unfortunately, the large gaps that can cause trouble and misinterpretation of cubic splined time series. Thus one should handle the analysis with care. (Panichi et al. 2018) showed that the integration time plays an important role in the stability investigation of this particular systems. Although, the initial conditions of the inner planet (Kepler$29 \mathrm{~b})$ are sitting well inside the regular domain of the $(a, e)$ stability map for shorter time, it turns out that the center of the resonance becomes unstable for longer integration. The initial conditions eventually appear at the border of the regular and chaotic domain falling into the sticky region, similarly to Kepler-36.

The results of the hypothesis tests based on the available data sets are shown in Figures 14 and 15 predict regular dynamics for the inner as well as for the outer planet. This is not in contradiction to previous findings bearing in mind that the time series is extremely short. It might present chaotic nature for longer time scales. Moreover, in case of Kepler-36 system the planets are closer to each other with an order of magnitude resulting in much stronger mutual gravitational perturbation and dynamical effect on shorter times. Thus, the different dynamical time scales can explain the more irregular outcome of the stability investigation of Kepler-36 from the same kind (length, noise, gaps) of data set.

\section{CONCLUSIONS}

In this paper we describe the stability of multiplanetary 760 systems based solely on astronomical observations. The 761 technique involves complex network analysis through phase 762 space reconstruction (time delay embedding) and recurrence 763 plot representation (Figure 3). The Sun-Jupiter-Saturn sys- 764 tem is used for pedagogical purposes. This well-known two- 765 planet configuration serves a large variety of motions (pe- 766 riodic, quasi-periodic, chaotic, sticky) what can also be ob- 767 served in extrasolar planetary systems. Having the knowl- 768 edge about the SJS dynamics (indicators MEGNO) we are 769 able to test the new strategy to obtain the stability and 770 explore the strength, weakness, and applicability of the 771 method. We have shown that complex network measures 772 (such as average path length, transitivity) applied in recur- 773 rence network (RN) framework characterize the system dy- 774 namics accurately. Moreover, our strategy copes with noisy 775 and non uniformly sampled time series as well. The whole 776 procedure has been applied in real exoplanetary systems. In 777 spite of short and sparse data sets the analyses match with 778 earlier stability studies of the same systems.

Although the long-term stability is the primary goal of 780
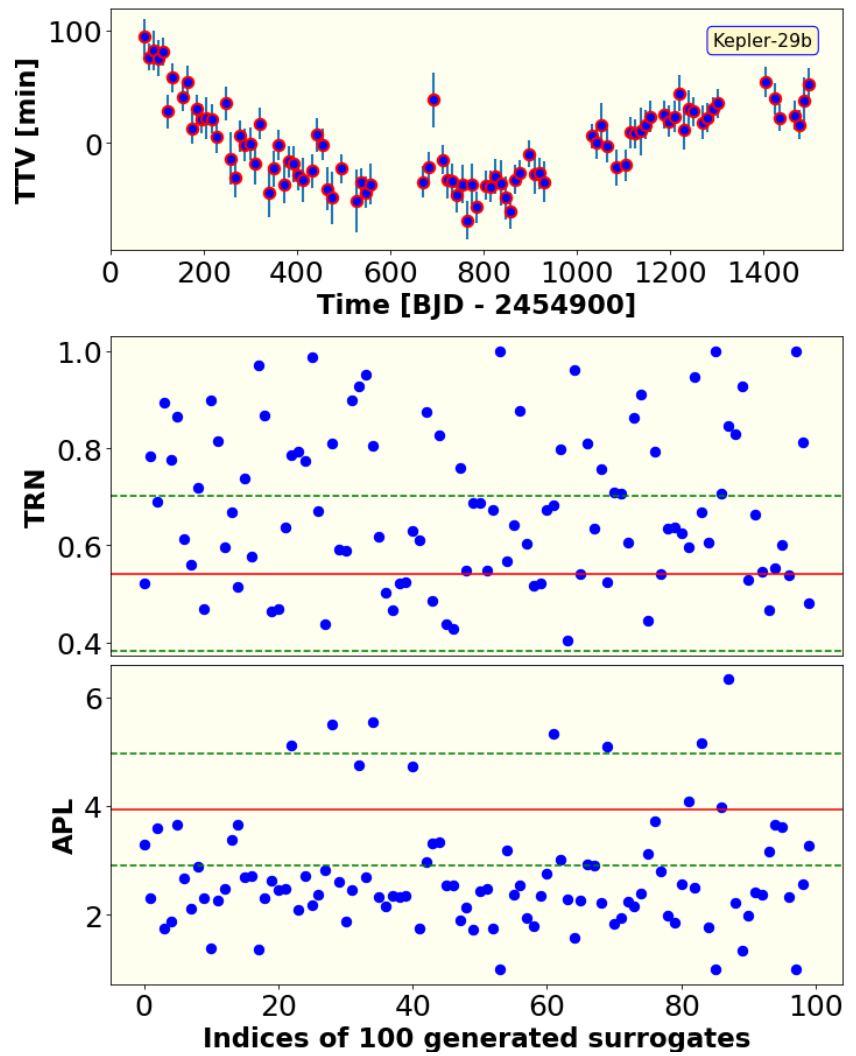

Figure 14. TTV Kepler-29b (missing data $32 \%$ ). Hypothesis test. $T R N=0.541, A P L=3.932, \rho=12.6$.

dynamical analysis it should be noted that the method we present is strictly based on the signal measured. It means no further temporal extension of the phase space trajectory is available, say for millions of orbital revolutions, like in the case of numerical integration. Numerical integration is , however, the conventional approach that uses the orbital parameters as initial conditions obtained from the best fitting planetary models. And then applying one of the chaos indicator methods to find long time behaviour of the system. Our technique describes, in contrast, the dynamical behaviour of reconstructed phase space trajectory based on the time frame of the available observations. Clearly, for the direct problem (i.e. numerical integration of the initial conditions), one can find different stability phenomenon for slightly modified initial conditions or for the same initial states but different evolution time. For example, high eccentricity motion often leads to escape from the system that occurs for sooner or later. Obviously, no chaos detecting method can obey such a situation properly. Furthermore, close to the border of resonances, where stickiness acts, the orbit might exhibit regular situation, however, after a time it detaches from that domain and behaves chaotically (or escapes). In this study we show how the method works for a certain time interval (1050 orbits for Jupiter sampled by 950 measured points) in case of RV and the same number of transits (950). We note that the method was tested for longer and shorter integration times. That is, the MEGNO map for the same (a,e) parameter plane has been calculated for different time intervals and, consequently, its structure was different, especially 

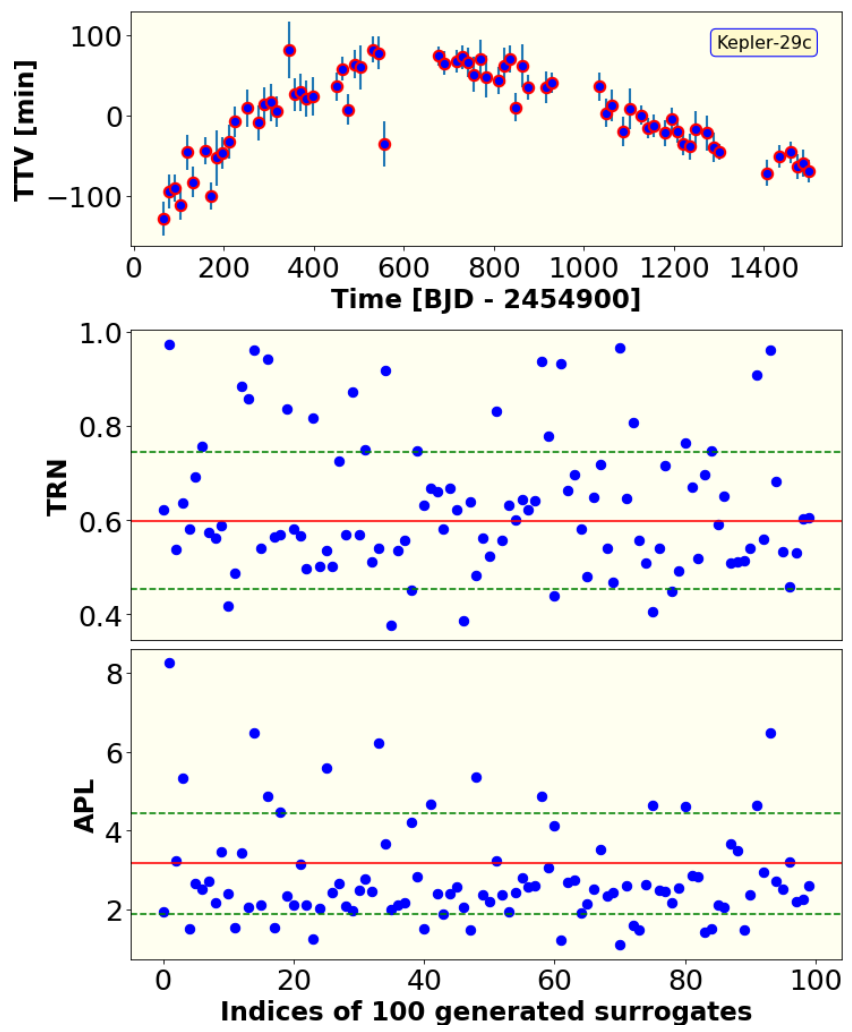

Figure 15. TTV Kepler-29c (missing data $36 \%$ ) and hypothesis test. $T R N=0.598, A P L=3.161, \rho=14.21$.

at MMRs. Nevertheless, NR measures were also capable in these situation to catch the distinct dynamical situations. $\quad{ }_{841}$

Let us draw a parallel between the direct numerical in- 842 tegration and RN method. The best fit planetary model per- 843 mits accurate initial parameters for further numerical inte- 844 gration. However, these initial conditions strongly depend 845 on the length of the time series as well as S/N. Fine tun- 846 ing the initial parameters by improving these circumstances 847 (follow-up observations) might lead to different kind of dy- ${ }_{84}$ namics than those emerging from the "obsolete" orbital el- 84 ements. Exactly the same situation holds for RN measures. 850 The more longer the data set, i.e. covers larger segments of 851 the dynamics, the precise the stability analysis. Indeed, no 852 extrapolation for the far future is needed and also the whole 853 procedure does not require neither the initial conditions nor 854 the system parameters and the equations of motion explic- 855 itly. All in all, what we can do with MEGNO for a given 856 integration time, RN measures do the same job for identical ${ }_{85}$ length of time series as well.

To our best knowledge, this study is the first step of ap- 859 plication of nonlinear time series analysis methods to obtain 860 stability of exoplanetary systems. Hence, there are many 861 ways to improve it. We mention some of them based on our experience so far.

- Length of the time series: Long-term stability analysis requires sufficiently long phase space trajectories to determine either the LCEs or other more efficient ${ }^{6}$ chaos detec-

6 More efficient means in this sense that the method can distin- tion quantities. This is also true for indirect methods as well. Fortunately, we can expect that the signals will be more populated and precise in the future.

- Combining RV-TTV-ASTROMETRY data: Various system information acquired by several observation techniques can describe distinct physical actions. The above mentioned observables give us different insights into the dynamics. Obviously, these are coupled since they come from the same system. The recurrence network method used in this work allows to mix different kind of measurements ${ }^{7}$ and with the help of this more information about the system dynamics can be obtained.

- Different surrogate algorithms: Surrogation is a widely used technique in time series analysis. In this study we performed the analysis by using the PPTS algorithm. However, there are other methods that are probably more sensitive, say, for the linear colored observational noise added to the system (Luo et al. 2005). Therefore, it is worth checking different surrogate generation methods to distinguish chaotic and regular motion in dynamical systems. Furthermore, we used only one static significance test (based on 100 surrogates which yields $99 \%$ level). One also might carry out more tests. In these situations the total probability of a false rejection can be different. To deal with such a scenario and how to modify the significance level we refer to the textbook (Kantz \& Schreiber 2003).

- Different embedding techniques: Phase space reconstruction is a must in RN analysis. Besides the classical time delay embedding there are various techniques that guarantee a successful trajectory reconstruction Lekscha \& Donner (2018). Moreover, one can also find alternative parameter estimation of delay embedding (Small \& Tse 2004; Hirata et al. 2006; Nichkawde 2013) as well as different kind of reconstruction methods (Hirata \& Aihara 2017; Uzal et al. 2011; Carroll 2018a; Lekscha \& Donner 2018) that can also be tested in dynamical astronomy.

- Other types of networks: Once we have the reconstructed phase space trajectory the natural choice is to use RN measures. However, other types of networks are suitable to explore the dynamical variability. Visibility graphs (Lacasa et al. 2008; Zou et al. 2014; Mutua et al. 2016) are promising candidates to use them as an alternative approach in planetary dynamics.

- Machine learning techniques: Many papers appeared recently about the attractor reconstruction and exploration of chaos in dynamical systems making use of machine learning techniques called reservoir computing (Pathak et al. 2017; Lu et al. 2018; Nakai \& Saiki 2018; Carroll 2018b). In this process the input data are the measured time series like in our present analysis. Moreover, the method could also solve the problem of surrogate analysis which is naturally encoded (as modified autonomous reservoir) in the mechanism they use. An additional method that might fit well to our purposes is convolutional networks of $2 \mathrm{D}$ image processing. A fresh paper (Hatami et al. 2018) proposed a strategy wherein a convolutional neural network (CNN) classifier has been

guish chaotic and regular motion from short (few thousands of orbital period) integration times.

7 This is true only when some basic criteria about the time series are fulfilled. See more about joint recurrence plots and crossrecurrence plots. (Marwan et al. 2007) 
applied to recurrence plots obtained from time series of dy- ${ }_{923}$ namical systems. They assert that CNN model works better ${ }_{924}$ for texture images (practically RPs) than other time series 925 classification schemes. These methods hold a significant po- ${ }^{926}$ tential to improve future dynamical modeling not only for ${ }^{927}$ planetary sciences but other fields of physics.

- High dimensional Hamiltonian problems: Not only for recurrence based analysis but for other network measures most of the analysis covers the well-known didactic examples such as dissipative Rössler, Lorenz, Hènon-Heiles systems or the classical Standard map as a Hamiltonian example. It would be extremely beneficial to examine how the network 935 measures behave in more complex phase space e.g. in high 936 dimensional Hamiltonian systems.

In summary, we believe that the method presented above can be used as a completion or prerequisite of dynamical analysis based on best fit planetary models followed by numerical N-body integration. Furthermore, recent efforts (Carter \& Agol 2013; Deck et al. 2014; Forgács-Dajka et al. ${ }_{943}$ 2018) show significant improvement in indirect stability and 944 dynamical analysis based on measured time series that also 945 supports our strategy.

\section{ACKNOWLEDGEMENTS}

This work was supported by the NKFIH Hungarian Grants K119993, PD121223. The support of Bolyai Research Fellowship is also acknowledged.

\section{REFERENCES}

Agol E., Deck K., 2016, ApJ, 818, 177

Agol E., Steffen J., Sari R., Clarkson W., 2005, MNRAS, 359, 567

Albert R., Barabási A.-L., 2002, Reviews of Modern Physics, 74, 47

Armstrong D. J., et al., 2015, A\&A, 582, A33

Barrat A., Weigt M., 2000, European Physical Journal B, 13, 547

Batygin K., Deck K. M., Holman M. J., 2015, AJ, 149, 167

Berndt D. J., Clifford J., 1994, in Proceedings of the 3rd International Conference on Knowledge Discovery and Data Mining. AAAIWS'94. AAAI Press, pp 359-370, http://dl.acm.org/ citation. $\mathrm{cfm}$ ? id $=3000850.3000887$

Boccaletti S., Latora V., Moreno Y., Chavez M., Hwang D.-U., 2006, Phys. Rep., 424, 175

Carrión A., Miralles R., 2016, in Webber Jr. C. L., Ioana C., Marwan N., eds, Springer Proceedings in Physics Vol. 180, Recurrence Plots and Their Quantifications: Expanding Horizon: Proceedings of the 6th International Symposium on Recurrence Plots. p. 91, doi:10.1007/978-3-319-29922-8_5

Carroll T. L., 2018a, Chaos, 28, 103117

Carroll T. L., 2018b, Phys. Rev. E, 98, 052209

Carter J. A., Agol E., 2013, ApJ, 765, 132

Cincotta P. M., Simó C., 2000, A\&AS, 147, 205

Deck K. M., Agol E., 2016, ApJ, 821, 96

Deck K. M., Holman M. J., Agol E., Carter J. A., Lissauer J. J., Ragozzine D., Winn J. N., 2012, ApJ, 755, 21

Deck K. M., Agol E., Holman M. J., NesvornÂAt'y D., NesvornÂt'y N., 2014, ApJ, 787, 132

Donges J. F., et al., 2015, Chaos, 25, 113101

Donner R. V., Zou Y., Donges J. F., Marwan N., Kurths J., 2010, 9 New Journal of Physics, 12, 033025

Donner R. V., Heitzig J., Donges J. F., Zou Y., Marwan N., 98 Kurths J., 2011, Eur. Phys. J. B, 84, 653
Eckmann J.-P., Oliffson Kamphorst S., Ruelle D., 1987, EPL (Europhysics Letters), 4, 973

Fabrycky D. C., 2010, in Seager S., ed., Exoplanets. pp 217-238

Fabrycky D. C., et al., 2012, ApJ, 750, 114

Fabrycky D. C., et al., 2014, ApJ, 790, 146

Foreman-Mackey D., Hogg D. W., Lang D., Goodman J., 2013, PASP, 125, 306

Forgács-Dajka E., Sándor Z., Érdi B., 2018, MNRAS, 477, 3383

Goździewski K., Migaszewski C., Panichi F., Szuszkiewicz E., 2016, MNRAS, 455, L104

Hadden S., Lithwick Y., 2016, ApJ, 828, 44

Hatami N., Gavet Y., Debayle J., 2018, in Tenth International Conference on Machine Vision (ICMV 2017). p. 106960Y, doi: $10.1117 / 12.2309486$

Hegger R., Kantz H., Schreiber T., 1999, Chaos, 9, 413

Hirata Y., Aihara K., 2017, Phys. Rev. E, 96, 032219

Hirata Y., Suzuki H., Aihara K., 2006, Phys. Rev. E, 74, 026202

Holczer T., et al., 2016, ApJS, 225, 9

Holman M. J., Murray N. W., 2005, Science, 307, 1288

Jontof-Hutter D., et al., 2016, ApJ, 820, 39

Kantz H., Schreiber T., 2003, Nonlinear Time Series Analysis. Cambridge University Press

Kovacs T., 2019, Chaos Fast Track, 29, 071105

Lacasa L., Luque B., Ballesteros F., Luque J., Nuño J. C., 2008, Proceedings of the National Academy of Science, 105, 4972

Laughlin G., Chambers J. E., 2001, ApJ, 551, L109

Laughlin G., Chambers J., 2006, ISSI Scientific Reports Series, 6, 233

Lekscha J., Donner R. V., 2018, Chaos, 28, 085702

Lu Z., Hunt B. R., Ott E., 2018, Chaos, 28, 061104

Luo X., Nakamura T., Small M., 2005, Phys. Rev. E, 71, 026230

Ma H.-G., Chong-Zhao H., 2006, Front. Electr. Electron. Eng. China, 1, 111

Mañé R., 1981, Lecture Notes in Mathematics, Berlin Springer Verlag, 898, 230

Marwan N., Kurths J., 2004, Cross Recurrence Plots and Their Applications, doi:10.1093/brain/aws023

Marwan N., Carmen Romano M., Thiel M., Kurths J., 2007, Physics Reports, 438, 237

Marwan N., Kurths J., Foerster S., 2015, Physics Letters A, 379, 894

Müller M., 2007, Dynamic Time Warping. Springer Berlin Heidelberg, Berlin, Heidelberg, doi:10.1007/978-3-540-74048-3_4

Mutua S., Gu C., Yang H., 2016, Chaos, 26, 053107

Nakai K., Saiki Y., 2018, Phys. Rev. E, 98, 023111

Nelson B. E., Ford E. B., Wright J. T., Fischer D. A., von Braun K., Howard A. W., Payne M. J., Dindar S., 2014, MNRAS, 441,442

Nesvorný D., Beaugé C., 2010, ApJ, 709, L44

Nesvorný D., Morbidelli A., 2008, ApJ, 688, 636

Newman M. E. J., 2003, SIAM Review, 45, 167

Ngamga E. J., Senthilkumar D. V., Prasad A., Parmananda P., Marwan N., Kurths J., 2012, Phys. Rev. E, 85, 1

Nichkawde C., 2013, Phys. Rev. E, 87, 022905

Ott E., 2002, Chaos in Dynamical System. Cambridge University Press, doi:10.2277/0521811961

Packard N. H., Crutchfield J. P., Farmer J. D., Shaw R. S., 1980, Physical Review Letters, 45, 712

Pál A., 2010, MNRAS, 409, 975

Panichi F., Goździewski K., Migaszewski C., Szuszkiewicz E., 2018, MNRAS, 478, 2480

Pathak J., Lu Z., Hunt B. R., Girvan M., Ott E., 2017, Chaos, 27,121102

Rein H., Tamayo D., 2015, MNRAS, 452, 376

Rivera E. J., Lissauer J. J., 2001, ApJ, 558, 392

Saussol B., Troubetzkoy S., Vaienti S., 2002, Journal of Statistical Physics, 106 
Schreiber T., Schmitz A., 2000, Physica D: Nonlinear Phenomena, 1037 142,346

Semmlow J. L., Griffel B., 2014, Biosignal and medical image processing. CRC Press

Shimada Y., Kimura T., Ikeguchi T., 2008, in , Artificial Neural Networks - ICANN 2008. Springer Berlin Heidelberg, Berlin, Heidelberg, pp 61-70, doi:10.1007/978-3-540-87536-9_7

Small M., Tse C. K., 2004, Physica D Nonlinear Phenomena, 194, 283

Small M., Yu D., Harrison R. G., 2001, Physical Review Letters, 87,188101

Steffen J. H., et al., 2012, MNRAS, 421, 2342

Stergiou N., 2016, Nonlinear analysis for human movement variability. CRC Press

Takens F., 1981, Lecture Notes in Mathematics, Berlin Springer Verlag, 898, 366

Tan X., Payne M. J., Lee M. H., Ford E. B., Howard A. W., Johnson J. A., Marcy G. W., Wright J. T., 2013, ApJ, 777, 1039 101

Tél T., Gruiz M., 2006, Chaotic Dynamics. Cambridge University ${ }_{1041}$ Press, doi:10.2277/0521839122

Theiler J., Prichard D., 1996, Physica D Nonlinear Phenomena, ${ }_{1043}{ }^{1042}$ 94, 221

Theiler J., Eubank S., Longtin A., Galdrikian B., Doyne Farmer ${ }^{1044}$ J., 1992, Physica D: Nonlinear Phenomena, 58, 77

Tsiganis K., Anastasiadis A., Varvoglis H., 2000, Chaos, Solitons \& Fractals, 11, 2281 016223

Veras D., Ford E. B., Payne M. J., 2011, ApJ, 727, 74

Xu X., Zhang J., Small M., 2008, Proceedings of the National Academy of Sciences of the United States of America, 105, 19601

Zou Y., Pazó D., Romano M. C., Thiel M., Kurths J., 2007, Phys. Rev. E, 76, 1

Zou Y., Donner R. V., Donges J. F., Marwan N., Kurths J., 2010, Chaos, 20, 043130

Zou Y., Donner R. V., Marwan N., Small M., Kurths J., 2014, Nonlinear Processes in Geophysics, 21, 1113

Zou Y., Donner R. V., Thiel M., Kurths J., 2016, Chaos, 26, 023120

\section{APPENDIX A: TIME DELAY EMBEDDING IN PRACTICE}

Let us consider a heuristic example for the delay reconstruc- ${ }^{1053}$ tion using synthetic measurements. The univariate data set, ${ }^{1054}$ containing 600 points, is given as

$x\left(t_{i}\right)=\left(\begin{array}{c}\# 1 \\ \# 2 \\ \# 3 \\ \vdots \\ \# 598 \\ \# 599 \\ \# 600\end{array}\right), \quad i=1 \ldots 600$

Next, choose the embedding parameters, for instance, 1066
Uzal L. C., Grinblat G. L., Verdes P. F., 2011, Phys. Rev. E, 84, 1046

$m=3, \tau=4$. Consequently, the size of the matrix $\mathbf{x}_{N}$ is $3 \times 592$ as it can be seen below

$\mathbf{x}_{N}=\left\{x\left(t_{i}-2 \cdot 4\right), x\left(t_{i}-4\right), x\left(t_{i}\right)\right\}=\left(\begin{array}{ccc}\# 9 & \# 5 & \# 1 \\ \# 10 & \# 6 & \# 2 \\ \# 11 & \# 7 & \# 3 \\ \vdots & \vdots & \vdots \\ \# 598 & \# 594 & \# 590 \\ \# 599 & \# 595 & \# 591 \\ \# 600 & \# 596 & \# 592 \\ \hline & \vdots & \vdots \\ & & \vdots \\ & \# 600 & \vdots \\ & & \# 600\end{array}\right)$.

Those elements that remain under the horizontal line are omitted. In this method the recovered signals are in rows as indicated in shaded box in $\mathbf{x}_{N}$, i.e. in our example the original time series elements $(\# 9, \# 5, \# 1)$ represents the first point of the reconstructed phase space trajectory in $m=3$ dimensions.

\section{APPENDIX B: RN MEASURES IN HIGH-DIMENSIONAL MAPS}

In this section we investigate how the $\mathrm{RN}$ measures vary when the dimension of the system is increasing. The main objective is to demonstrate that transitivity $(T R N)$ might have lower values for ordered motion than for chaotic ones in higher dimensions. As a model for our study we consider the $4 \mathrm{D}$ and $18 \mathrm{D}$ symplectic maps consisting of 2 and 9 coupled standard maps. The general formalism of symplectic coupling of $N$ symplectic 2D maps is the following

$$
\begin{aligned}
& \Theta_{i}^{n+1}=\Theta_{i}^{n}+p_{i}^{n+1}, \\
& p_{i}^{n+1}=p_{i}^{n}+K \sin \Theta_{i}^{n}-\sum_{j=1}^{N} B_{i, j} \sin \left[\Theta_{j}^{n}-\Theta_{i}^{n}\right],
\end{aligned}
$$

政

where $K$ is the nonlinearity parameter and $B_{i, j}$ is the coupling strength. The coupling on the $i$-th map is a perturbation in $p_{i}$ and the full coupling is symplectic provided $B_{i, j}=B_{j, i}=B$. System (B1) is a typical chaotic conservative system with mixed phase space. To show the behaviour of RN measures we fix the parameters $K=1.5$ and $B=0.0,0.005$, and 0.05 . Furthermore, the initial conditions are the following $\left(\Theta_{10}, p_{10}\right)=\left(3.241, p_{10}\right)$ where $p_{10} \in[0,2 \pi]$, $\left(\Theta_{i 0}, p_{i 0}\right)=(3.24,0.05), i=2 \ldots 9$.

Phase portraits for different $B$ parameters and $N=2$ are shown in Figure B1 bottom row. The classical structure is depicted when $B=0$, i.e. the two maps are decoupled. Invariant curves fade in higher dimension since in this case $\left(\Theta_{1}, p_{1}\right)$ section is a projection of the $4 \mathrm{D}$ phase space. Indicating the stability we overplot the Lyapunov exponents along the line of initial conditions. One can observe that the main islands remain stable and only the small stability regions become unstable for larger $B$.

Considering the recurrence network measures the 2D 


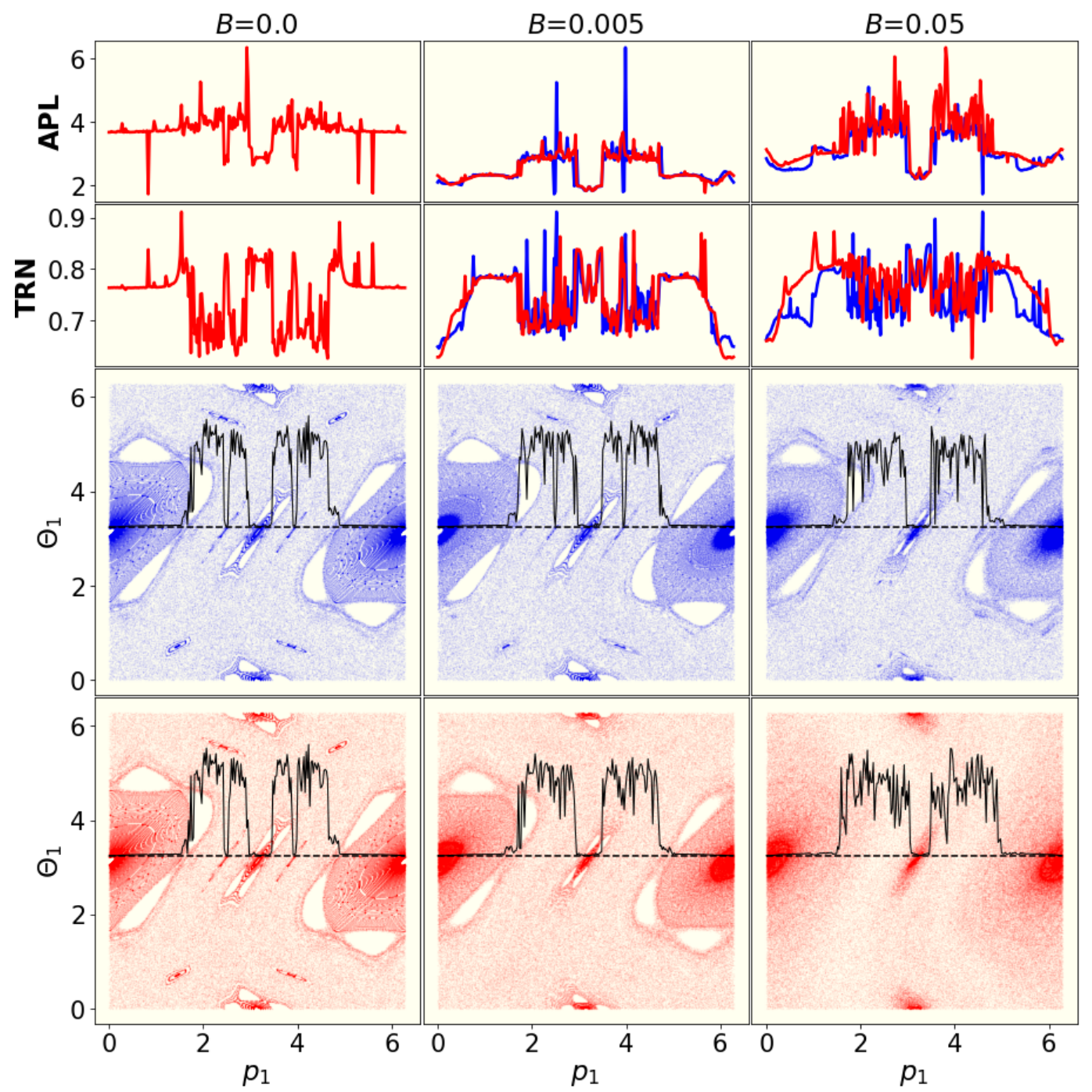

Figure B1. RN measures $A P L$ (top) and $T R N$ (middle) for coupled $4 \mathrm{D}$ standard map $(N=2)$. The larger the $B$, the stronger the coupling. The pattern specifies the lower TRN for regular motion when the extended phase space becomes more significant. Bottom: Phase portraits of $\left(\Theta_{1}, p_{1}\right)$ sections. As expected the well-known embedded phase space structure vanishes in higher dimension. Note that the LCE curves (purple) does not show the real value, they are enlarged (by a factor of 10) for better visualization. Red part: now $N=9$, that is, the phase space has 18 dimensions.

map serves what we expect from literature (Zou et al. 2016). 1085 That is, smaller value of APL but larger value of TRN for 1086 periodic motion. In turn, when the phase space is extended 1087 the value of $T R N$ starts to decrease at regular domains. The higher the coupling, the larger the $T R N$ 's decay, $A P L$ shows the same characteristic. In other words, the large stability is- 1088 land keeps its stability (the LCEs remain zero) while the net- 1089 work measure $T R N$ turns to be low. Similar behaviour can be 1090 seen in (Marwan et al. 2015) when they found smaller TRN 1091 for stable motion $(L C E=0)$ than for unstable in Lorenz96 1092 model. Although, the Lorenz96 system is a high dimensional continuous dynamical model, the explanation of the phenomenon can be the same. Namely, in higher dimensions, 1093 periodic orbits are not confined to a finite part of the phase 1094 space but can sweep a large domain that is comparable with 1095 chaotic realm in size. Consequently, the clustering, and also 1096 the transitivity, due to the divergence of the trajectories is 1097 not so efficient in resulting in smaller values even in the case 1098 of periodic motion. This fact might clarify the lower TRN values for regular dynamics in the three body problem, Section 3.3 Fig. 6.

For higher dimensional phase space, for instance $N=9$, the tendency is similar. As one can see the essential difference between the uncoupled and coupled scenarios follows our former observation. The phase portrait get more fuzzy and with this the RN measures drop off for regular motion.

Based on these findings we believe in the results presented in Fig. 6(d) wherein smaller TRNs describe the regular dynamics. The precise study of RQA and RN description of high-dimensional Hamiltonian systems (either continuous or discrete) is crucial and must be carried out carefully in the future. To our best knowledge it has not been done yet. We postpone this work elsewhere. 


\section{APPENDIX C: DYNAMIC TIME WARPING}

As we have seen, the noise radius $\rho$ is a pivotal parameter in pseudo-periodic twin surrogates algorithm (PPTS), see Section 3.5.1. Its value tunes the PPTS which means if $\rho$ is too large the generated surrogate will be a sequence of random values. While, in contrast, if the value of $\rho$ is too small, the produces time series is identical to the original one. What we need, therefore, is a method that gives a suitable noise radius which is not too large and not too small. In other words, we want to generate a time series that contains some dynamics noise, nevertheless, it is similar to the original signal compared by naked eyes.

In this section we propose the method of dynamics time warping (DTW) that is suitable to quantify the similarity of two time series (Berndt \& Clifford 1994). Without going into the details we present some of the basic feature of DTW and then present how it works in case of PPTS algorithm.

Comparing two sequences, possible with different length, one needs a local cost measure. This measure is typically small if the two signals are similar to each other, and large otherwise. Then one can construct the cost matrix that defines the local cost measure between each pair of points of two signals. As a final step the task is to find an alignment between of the two time series that minimizes the overall cost. For a more precise mathematical formulation of DTW see reference (Müller 2007).

Based on the algorithm in Section 3.5.1 a large number (250) of PPTSs has been generated over several orders of magnitude of $\rho$. Then we stored the actual value of DTW. Small DTW characterizes minor difference between the original signal and the generated surrogate while relative large value reveals significant contrast between them. See Figure $\mathrm{C} 1$ for specifying the noise radius in two different dynamical regime.

For practical purposes we accept the appropriate noise radius when the DTW curve (black solid line) leaves the 1 standard deviation defined by the first $20 \%$ of data points (green dashed line). Using this rule is a must because the first part of the plot, i.e. the DTW for small noise radii, might fluctuate in a great way, especially in strongly chaotic ${ }^{1165}$ cases.

The upper panel of Fig. C1 portrays the relationship between $D T W$ and noise radius for the regular orbit originating at $\left(a_{\text {Saturn }}, e_{\text {Saturn }}\right)=(7.2,0.02)$. The two well separated values of $D T W$ indicate the role of the noise radius explained above. The smaller value of $D T W(\rho \lesssim 0.1)$ characterizes that the two time series are similar while the right tale of the plot $(\rho \gtrsim 3)$ denotes when the surrogate and the original signal are completely different. The noise radius should be chosen from the rest part of the curve. The blue dashed line is placed to the value of $\rho=0.075$ where $D T W$ starts to diverge from its mean value $\left(\sim 5 \times 10^{4}\right)$ calculated from the first $20 \%$ of the data points.

The lower panel shows $D T W$ vs. noise radius for the chaotic orbit starting at $\left(a_{\text {Saturn }}, e_{\text {Saturn }}\right)=(8.0,0.2)$. The main structure of the plot matches the upper one. However, the $D T W$ values are larger by an order of magnitude. The noise radius corresponding to the 1 standard deviation limit ( $\rho=0.13)$ is marked by the vertical blue dashed line.
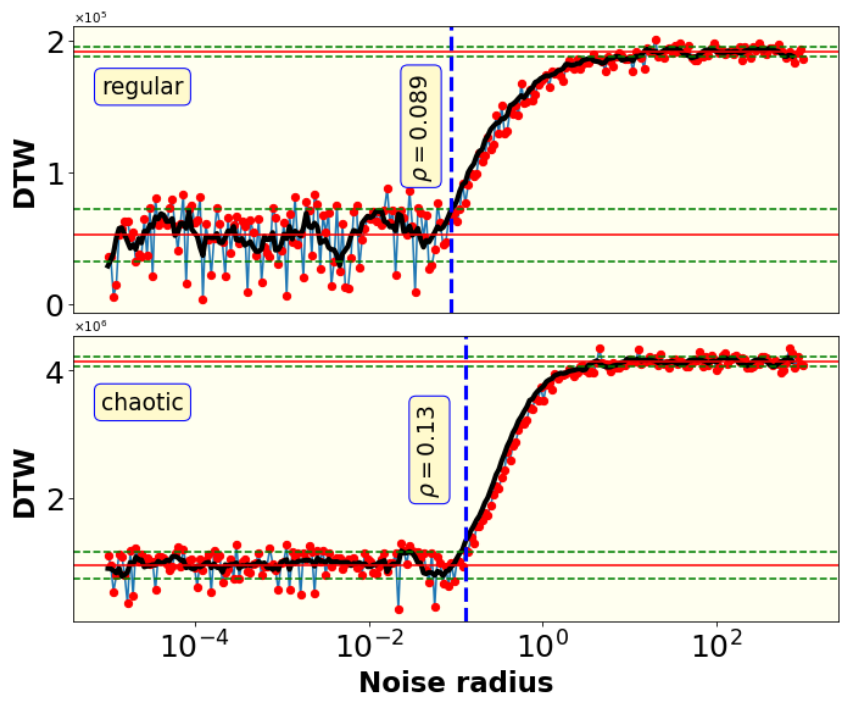

Figure C1. Dynamic time warping vs. noise radius. Top: regular dynamics. Bottom: chaotic dynamics. The reference signal in both cases is Jupiter's TTV, see Fig. 9(a) and (b). Different scale of dynamical noise in PPT surrogate algorithm yields distinct features in resulting surrogate time series. The intermediate values present a suitable noise radius to generate PPTSs having the same intracycle dynamics (but vanishing the intercycle structures) as the original data set. Red solid lines correspond to mean DTW value of first and last $20 \%$ of data points, respectively. The green dashed lines mark the \pm 1 standard deviation of the same segments of data. The thick black curve represents a smoothing of the original 250 data points by a moving average (window size is 5$)$.

Making use of DTW we are able to set the appropriate value of noise radius $(\rho)$ in PPTS algorithm, see Eq. 9. We should however emphasize that the choice of 1 sigma limit is empirical and further investigation is needed how to suit more precisely the DTW algorithm to find the best $\rho$.

This paper has been typeset from a $\mathrm{T}_{\mathrm{E}} \mathrm{X} / \mathrm{LAT}_{\mathrm{E}} \mathrm{X}$ file prepared by the author. 